\title{
LOCAL FUNCTIONAL EQUATIONS FOR SUBMODULE ZETA FUNCTIONS ASSOCIATED TO NILPOTENT ALGEBRAS OF ENDOMORPHISMS
}

\author{
CHRISTOPHER VOLL
}

\begin{abstract}
We give a sufficient criterion for generic local functional equations for submodule zeta functions associated to nilpotent algebras of endomorphisms defined over number fields. This allows us, in particular, to prove various conjectures on such functional equations for ideal zeta functions of nilpotent Lie lattices. Via the Mal'cev correspondence, these results have corollaries pertaining to zeta functions enumerating normal subgroups of finite index in finitely generated nilpotent groups, most notably finitely generated free nilpotent groups of any given class.
\end{abstract}

- In memory of Anton Evseev.

\section{INTRODUCTION}

1.1. Submodule zeta functions for nilpotent associative algebras of endomorphisms. Let $R$ be the ring of integers $\mathcal{O}$ of a number field or a compact discrete valuation ring, such as the completion $\mathcal{O}_{\mathfrak{p}}$ of such a ring $\mathcal{O}$ at a nonzero prime ideal $\mathfrak{p}$ of $\mathcal{O}$ (in characteristic zero) or a formal power series ring $(\mathcal{O} / \mathfrak{p}) \llbracket T \rrbracket$ (in characteristic $p>0$ ). Let $\mathcal{L}$ be a free $R$-module of finite rank $n$ and $\mathcal{E}$ be a (not necessarily unital) subalgebra of the associative $R$-algebra $\operatorname{End}_{R}(\mathcal{L})$. For $m \in \mathbb{N}$, let

$$
a_{m}(\mathcal{E} \curvearrowright \mathcal{L})=\#\left\{H \leq \mathcal{L} \mid H \text { is an }\left(\mathcal{E}+R \operatorname{id}_{\mathcal{L}}\right) \text {-submodule of } \mathcal{L} \text { with }|\mathcal{L}: H|=m\right\} .
$$

We define the submodule zeta function of $\mathcal{E}$ acting on $\mathcal{L}$ as the formal Dirichlet generating function

$$
\zeta_{\mathcal{E} \curvearrowright \mathcal{L}}(s)=\sum_{m=1}^{\infty} a_{m}(\mathcal{E} \curvearrowright \mathcal{L}) m^{-s},
$$

where $s$ is a complex variable; cf. [23, Definition 2.1 (ii)]. The submodule zeta function $\zeta_{\mathcal{E} \curvearrowright \mathcal{L}}(s)$ may be viewed as a (non-unital) analogue of Solomon's zeta function; see [29]. Assume now that $R$ is the ring of integers $\mathcal{O}$ of a number field. Then $\zeta_{\mathcal{E} \curvearrowright \mathcal{L}}(s)$ satisfies the Euler product

$$
\zeta_{\mathcal{E} \curvearrowright \mathcal{L}}(s)=\prod_{\mathfrak{p}} \zeta_{\mathcal{E}\left(\mathcal{O}_{\mathfrak{p}}\right) \curvearrowright \mathcal{L}\left(\mathcal{O}_{\mathfrak{p}}\right)}(s)
$$

where the product ranges over the nonzero prime ideals of $\mathcal{O}$ and $\mathcal{E}\left(\mathcal{O}_{\mathfrak{p}}\right):=\mathcal{E} \otimes_{\mathcal{O}} \mathcal{O}_{\mathfrak{p}}$ and $\mathcal{L}\left(\mathcal{O}_{\mathfrak{p}}\right):=\mathcal{L} \otimes_{\mathcal{O}} \mathcal{O}_{\mathfrak{p}}$, regarded as an $\mathcal{O}_{\mathfrak{p}}$-algebra and $\mathcal{O}_{\mathfrak{p}}$-module, respectively. It follows from well-known results expressing counting functions such as $\zeta_{\mathcal{E}\left(\mathcal{O}_{\mathfrak{p}}\right) \curvearrowright \mathcal{L}\left(\mathcal{O}_{\mathfrak{p}}\right)}(s)$ in terms of $\mathfrak{p}$-adic integrals that each of the Euler factors is a rational function in $|\mathcal{O}: \mathfrak{p}|^{-s}$; see, for instance, [16] for the case $\mathcal{O}=\mathbb{Z}$ and, taken together, [17, 11] for the general case.

Date: September 24, 2018.

2000 Mathematics Subject Classification. 11M41, 20E07, 11S40, 16W20.

Key words and phrases. Submodule zeta functions, ideal zeta functions, nilpotent associative algebras of endomorphisms, finitely generated nilpotent groups, local functional equations. 
Assume now that $\mathcal{E}$ is nilpotent. The main objective of this paper is to establish in this case, under suitable conditions, functional equations for $\mathfrak{p}$-adic submodule zeta functions occurring as generic factors in Euler products of the form (1.1); see Theorem 1.2.

Prominent examples of submodule zeta functions of nilpotent associative algebras of endomorphisms are ideal zeta functions of nilpotent Lie lattices, which we now recall. Let $\mathcal{L}$ be an $\mathcal{O}$-Lie lattice, i.e. a free and finitely generated $\mathcal{O}$-module of finite rank $n$ equipped with an antisymmetric bi-additive form (or "Lie bracket") satisfying the Jacobi identity. By a Lie ring we mean a $\mathbb{Z}$-Lie lattice. For $m \in \mathbb{N}$, we write $a_{m}(\mathcal{L})=\#\left\{H \triangleleft_{\mathcal{O}} \mathcal{L}|| \mathcal{L}: H \mid=m\right\}$ for the number of $\mathcal{O}$-ideals of $\mathcal{L}$ of index $m$ in $\mathcal{L}$. The ideal zeta function of $\mathcal{L}$ is the Dirichlet generating series

$$
\zeta_{\mathcal{L}}^{\triangleleft}(s)=\sum_{m=1}^{\infty} a_{m}(\mathcal{L}) m^{-s} ;
$$

cf. [16, Section 3]. It fits into the setup from above by considering the associative subalgebra $\mathcal{E} \subseteq \operatorname{End}_{\mathcal{O}}(\mathcal{L})$ generated by $\operatorname{ad}(\mathcal{L}) ;$ clearly $a_{m}(\mathcal{L})=a_{m}(\mathcal{E} \curvearrowright \mathcal{L})$. The Euler product (1.1) takes the form

$$
\zeta_{\mathcal{L}}^{\triangleleft}(s)=\prod_{\mathfrak{p}} \zeta_{\mathcal{L}\left(\mathcal{O}_{\mathfrak{p}}\right)}^{\triangleleft}(s),
$$

where, for each prime ideal $\mathfrak{p}$, the Euler factor $\zeta_{\mathcal{L}\left(\mathcal{O}_{\mathfrak{p}}\right)}^{\triangleleft}(s)$ enumerates the $\mathcal{O}_{\mathfrak{p}}$-ideals of $\mathcal{L}\left(\mathcal{O}_{\mathfrak{p}}\right)$ of finite additive index in $\mathcal{L}\left(\mathcal{O}_{\mathfrak{p}}\right)$.

Returning to general nilpotent associative algebras of endomorphisms $\mathcal{E} \subseteq \operatorname{End}_{\mathcal{O}}(\mathcal{L})$ we define $\mathcal{O}$-submodules $Z_{i}$ of $\mathcal{L}$, for $i \in \mathbb{N}_{0}$, by setting $Z_{0}=\{0\}$ and

$$
Z_{i+1} / Z_{i}=\operatorname{Cent}_{\mathcal{E}}\left(\mathcal{L} / Z_{i}\right):=\left\{x+Z_{i} \in \mathcal{L} / Z_{i} \mid x \mathcal{E} \subseteq Z_{i}\right\}
$$

for $i>0$. As $\mathcal{E}$ is nilpotent there exists $i \in \mathbb{N}$ such that $Z_{i}=\mathcal{L}$; cf. [18, Chapter 2, Section II]. We set

$$
c=c(\mathcal{L}, \mathcal{E})=\min \left\{i \in \mathbb{N}_{0} \mid Z_{i}=\mathcal{L}\right\} .
$$

(If $\mathcal{L}$ is a nilpotent $\mathcal{O}$-Lie lattice and $\mathcal{E}$ is the associative subalgebra generated by $\operatorname{ad}(\mathcal{L})$, then $\left(Z_{i}\right)_{i=0}^{c}$ is just the upper central series of $\mathcal{L}$ and $c$ is the nilpotency class of $\mathcal{L}$.)

In this paper, we consider pairs $(\mathcal{L}, \mathcal{E})$ satisfying the following assumption.

Assumption 1.1. There exist free $\mathcal{O}$-submodules $\mathcal{L}_{1}, \ldots, \mathcal{L}_{c}$ of $\mathcal{L}$ such that

$$
Z_{i}=\bigoplus_{j>c-i} \mathcal{L}_{j}
$$

for $i=1, \ldots, c$. Note that, in particular,

$$
\mathcal{L}=\mathcal{L}_{1} \oplus \cdots \oplus \mathcal{L}_{c}
$$

(direct sum of $\mathcal{O}$-modules). We also set $\mathcal{L}_{0}=\mathcal{L}_{c+1}=\{0\}$.

Remark 1.1. Assumption 1.1 is only made for notational convenience. It is automatically satisfied if $\mathcal{O}$ is a unique factorization domain (e.g. $\mathbb{Z}$ ). In general, the "centralizers" $Z_{j}$ will be isolated in $\mathcal{L}$ (viz. the factor modules $\mathcal{L} / Z_{j}$ will be torsion-free), but may not allow complements. This may be mitigated by localizing $\mathcal{L}$ at a finite set of prime ideals of $\mathcal{O}$ or - by the general theory of finitely generated modules over Dedekind domains - by passing to a suitable finite index $\mathcal{O}$-submodule of $\mathcal{L}$; cf., for instance, the discussion in 32 , Section 2.3]. In any case, only finitely many of the Euler factors in (1.1) are affected. As we are only looking to prove results for all but finitely many of these, making Assumption 1.1 means no loss of generality.

Our main results concern local submodule zeta functions associated to general nilpotent algebras $\mathcal{E}$ which satisfy the following condition. 
Condition 1.1. The nilpotent associative algebra $\mathcal{E} \subseteq \operatorname{End}_{\mathcal{O}}(\mathcal{L})$ is generated by elements $c_{1}, \ldots, c_{d}$ such that, for all $k=1, \ldots, d$ and $j=1, \ldots, c$,

$$
\mathcal{L}_{j} c_{k} \subseteq \mathcal{L}_{j+1} .
$$

For a matrix version of this condition, see Condition 2.1.

Given a nonzero prime ideal $\mathfrak{p}$ of $\mathcal{O}$ we write $q$ for the cardinality of the residue field $\mathcal{O} / \mathfrak{p}$. The paper's main result establishes, in particular, functional equations upon inversion of $q$ for all but finitely many of the Euler factors in (1.1) in case that $(\mathcal{L}, \mathcal{E})$ satisfies Condition 1.1. For $i \in\{0,1, \ldots, c\}$ we write

$$
N_{i}=\operatorname{rk}_{\mathcal{O}} \bigoplus_{j \leq c-i} \mathcal{L}_{j}=\operatorname{rk}_{\mathcal{O}}\left(\mathcal{L} / Z_{i}\right),
$$

noting that $N_{0}=n=\operatorname{rk}_{\mathcal{O}} \mathcal{L}$ and $N_{c}=0$. Throughout this paper, by a finite extension of a local ring of the form $\mathcal{O}_{\mathfrak{p}}$ we mean the ring of integers of a finite extension of the local field of fractions of $\mathcal{O}_{\mathfrak{p}}$.

Theorem 1.2. Assume that $\mathcal{E} \subseteq \operatorname{End}_{\mathcal{O}}(\mathcal{L})$ satisfies Condition 1.1. Then, for almost all prime ideals $\mathfrak{p}$ of $\mathcal{O}$ and all finite extensions $\mathfrak{O}$ of $\mathcal{O}_{\mathfrak{p}}$, with residue field cardinality $q^{f}$, say, the following functional equation holds:

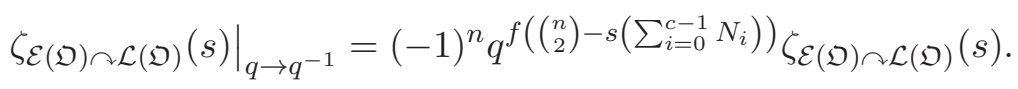

Whilst the local submodule zeta functions encountered in Euler products such as (1.1) are defined in terms of local rings of characteristic zero, they have counterparts in sufficiently large positive characteristic $p$. More precisely, expressing the relevant submodule zeta functions as p-adic integrals - be it cone integrals in the vain of [11] ((cf. [23, Theorem 5.16])) or the ones deployed in Section 2.1 - and employing a version of the transfer principle for such integrals (for instance [6. Theorem 9.2.4]), yields the following: for almost all $\mathfrak{p}$ and all finite extensions $\mathfrak{O}$ of $\mathcal{O}_{\mathfrak{p}}$, with maximal ideal $\mathfrak{P}$, say, setting $\mathcal{E}((\mathfrak{O} / \mathfrak{P}) \llbracket T \rrbracket)=\mathcal{E} \otimes_{\mathcal{O}}(\mathfrak{O} / \mathfrak{P}) \llbracket T \rrbracket$ and $\mathcal{L}((\mathfrak{O} / \mathfrak{P}) \llbracket T \rrbracket)=\mathcal{L} \otimes_{\mathcal{O}}(\mathfrak{O} / \mathfrak{P}) \llbracket T \rrbracket$, one has

$$
\zeta_{\mathcal{E}(\mathfrak{O}) \curvearrowright \mathcal{L}(\mathfrak{O})}(s)=\zeta_{\mathcal{E}((\mathfrak{O} / \mathfrak{P}) \llbracket T \rrbracket) \curvearrowright \mathcal{L}((\mathfrak{O} / \mathfrak{P}) \llbracket T \rrbracket)}(s) .
$$

The following is thus an immediate consequence of Theorem 1.2

Corollary 1.3. For almost all prime ideals $\mathfrak{p}$ of $\mathcal{O}$ and all finite extensions $\mathfrak{O}$ of $\mathcal{O}_{\mathfrak{p}}$, with maximal ideal $\mathfrak{P}$ and residue field cardinality $|\mathfrak{O} / \mathfrak{P}|=q^{f}$, say, the following functional equation holds:

$$
\left.\zeta_{\mathcal{E}((\mathfrak{O} / \mathfrak{P}) \llbracket T \rrbracket) \curvearrowright \mathcal{L}((\mathfrak{O} / \mathfrak{P}) \llbracket T \rrbracket)}(s)\right|_{q \rightarrow q^{-1}}=(-1)^{n} q^{f\left(\left(\begin{array}{l}
n \\
2
\end{array}\right)-s\left(\sum_{i=0}^{c-1} N_{i}\right)\right)} \zeta_{\mathcal{E}((\mathfrak{O} / \mathfrak{P}) \llbracket T \rrbracket) \curvearrowright \mathcal{L}((\mathfrak{O} / \mathfrak{P}) \llbracket T \rrbracket)}(s) .
$$

(We are grateful to an anonymous referee for asking about characteristic $p$ and to Raf Cluckers for directing us to modern versions of the transfer principle.)

Example 1.4. For $c=1$, Condition 1.1 is trivially satisfied as $\mathcal{E}=\{0\}$. Theorem 1.2 and Corollary 1.3 follow, in this case, by inspection of the classical formula, valid for any compact discrete valuation ring $\mathfrak{o}$ with residue field cardinality $q$, say,

$$
\zeta_{\{0\} \curvearrowright \mathfrak{o}^{n}}(s)=\frac{1}{\left(1-q^{-s}\right)\left(1-q^{1-s}\right) \ldots\left(1-q^{n-1-s}\right)}
$$

for the Dirichlet generating series enumerating all finite index submodules of $\mathfrak{o}^{n}$; see, for instance, [16, Proposition 1.1]. 
Remark 1.5. Whilst we cannot make any quantitative statements on the finite set of prime ideals of $\mathcal{O}$ to be excluded in Theorem 1.2, there are examples illustrating that this set of "bad primes" is not empty in general. We note that the assertion of Theorem 1.2 is uniform under global base extension in the sense that the possible exceptions to the Euler factors' functional equations are determined already by $(\mathcal{L}, \mathcal{E})$.

The necessity to disregard finitely many residue characteristics is one reason why, Corollary 1.3 notwithstanding, our exposition will for the most part concentrate on local submodule zeta functions in characteristic zero. Another motivation to focus on submodule zeta functions defined over number fields (as opposed, say, to global function fields) comes from the group-theoretic application to ideal zeta functions of finitely generated nilpotent groups; cf. Section 1.2 .

Remark 1.6. In the special case of ideal zeta functions associated to (not necessarily nilpotent) Lie rings, viz. $\mathbb{Z}$-Lie lattices, some of the identities (1.4) were established in 10 , Theorem 5.4]. Indeed, this result shows that the ideal zeta functions $\zeta_{\mathcal{L}\left(\mathbb{Z}_{p}\right)}(s)$ and $\zeta_{\mathcal{L}\left(\mathbb{F}_{p} \llbracket T \rrbracket\right)}^{\triangleleft}(s)$ of the $\mathbb{Z}_{p^{-}}$resp. $\mathbb{F}_{p} \llbracket T \rrbracket$-algebras obtained by tensoring a given Lie ring $\mathcal{L}$ with $\mathbb{Z}_{p}$ resp. $\mathbb{F}_{p} \llbracket T \rrbracket$ coincide for large $p$. The theorem rests on [10, Proposition 5.7], an instance of the Ax-Kochen-Ershov principle, attributed in [10 to a remark in 20], which establishes identities between certain definable integrals over $\mathbb{Z}_{p}$ resp. $\mathbb{F}_{p} \llbracket T \rrbracket$ for large $p$. Neither [10] nor [20], however, discuss the validity of the established identities after local ring extensions, let alone in a uniform manner.

The transfer principle as established by Cluckers-Loeser in [6] vastly generalizes earlier work, including the Ax-Kochen-Ershov principle. It allows, in particular, for the treatment of more complex classes of (motivic and) p-integrals but also - and most relevantly for the applications in the current paper -, cross-characteristic comparisons which are valid uniformly for local ring extensions, provided the residue characteristic is sufficiently large. This extra dimension of uniformity under local ring extensions offered by modern versions of the transfer principle is reflected in equations (1.4).

Remark 1.7. In general, the operation $q \rightarrow q^{-1}$ means the following. Given a finite extension $\mathfrak{O}$ of $\mathcal{O}_{\mathfrak{p}}$, write $\mathfrak{P}$ for the maximal ideal of $\mathfrak{O}$. Thus $|\mathfrak{O} / \mathfrak{P}|=q^{f}$. Our proof of Theorem 1.2 will show that, excluding finitely many $\mathfrak{p}$, the zeta function $\zeta_{\mathcal{E}(\mathfrak{O}) \curvearrowright \mathcal{L}(\mathfrak{O})}(s)$ may be written as a finite sum of the form

$$
\sum_{i=1}^{M}\left|\overline{V_{i}}(\mathfrak{O} / \mathfrak{P})\right| W_{i}\left(q^{f}, q^{-f s}\right),
$$

where the $\left|\overline{V_{i}}(\mathfrak{O} / \mathfrak{P})\right|$ denote the numbers of $\mathfrak{O} / \mathfrak{P}$-rational points of the reductions modulo $\mathfrak{p}$ of $\mathcal{O}$-defined smooth projective algebraic varieties $V_{i}$ and rational functions $W_{i}(X, Y) \in$ $\mathbb{Q}(X, Y)$. By the Weil conjectures, the numbers $\left|\overline{V_{i}}(\mathfrak{O} / \mathfrak{P})\right|$ may be written as alternating sums of Frobenius eigenvalues. By $q \rightarrow q^{-1}$ we mean the operation of inverting these eigenvalues (cf. [1, eqs. (4.10) and (4.11)]) and, of course, evaluating $W_{i}$ at $\left(q^{-f}, q^{f s}\right)$. If the reductions $\overline{V_{i}}$ are smooth (i.e. the $V_{i}$ have good reduction modulo $\mathfrak{p}$ ), then Poincaré duality for étale cohomology entails symmetries among the Frobenius eigenvalues which imply that $\left|\overline{V_{i}}(\mathfrak{O} / \mathfrak{P})\right|_{q \rightarrow q^{-1}}=q^{-f \operatorname{dim} V_{i}}\left|\overline{V_{i}}(\mathfrak{O} / \mathfrak{P})\right|$. In the special case that $\left|\overline{V_{i}}(\mathfrak{O} / \mathfrak{P})\right|$ is given by a polynomial $F_{V_{i}} \in \mathbb{Z}[X]$ in the residue field cardinality $q^{f}$, this amounts to the palindromic symmetry $F_{V_{i}}\left(X^{-1}\right)=X^{-\operatorname{dim} V_{i}} F_{V_{i}}(X)$. The rational functions $W_{i}$ admit a common denominator of the form $\prod_{j=1}^{r}\left(1-X^{a_{j}} Y^{b_{j}}\right)$ for $a_{j} \in \mathbb{N}_{0}, b_{j} \in \mathbb{N}, j \in\{1, \ldots, r\}$. That the functional equation (1.3) does not depend on the chosen formula (1.6) is shown 
in [21, Section 4]. By [21, Corollary 4.2], it suffices to show (1.3) for $\mathfrak{O}=\mathcal{O}_{\mathfrak{p}}$ for all but finitely many $\mathfrak{p}$.

Remark 1.8. Condition 1.1 is satisfied if $\mathcal{E}$ is cyclic (i.e. one may choose $d=1$; cf. Section 5.1) or if $\mathcal{E}^{2}=0$ (i.e. $c \leq 2$ ). Moreover, it is stable under taking direct products and (certain) central quotients: If $\mathcal{E}_{1} \subseteq \operatorname{End}_{\mathcal{O}}\left(\mathcal{L}_{1}\right)$ and $\mathcal{E}_{2} \subseteq \operatorname{End}_{\mathcal{O}}\left(\mathcal{L}_{2}\right)$ both satisfy Condition 1.1, then so does $\mathcal{E}_{1} \times \mathcal{E}_{2} \subseteq \operatorname{End}_{\mathcal{O}}\left(\mathcal{L}_{1} \oplus \mathcal{L}_{2}\right)$. If $\mathcal{E} \subseteq \operatorname{End}_{\mathcal{O}}(\mathcal{L})$ satisfies Condition 1.1 and $M \leq Z_{1}=\mathcal{L}_{c}$ is an isolated central $\mathcal{O}$-submodule admitting a complement in $\mathcal{L}_{c}$, then the induced algebra of endomorphisms $\overline{\mathcal{E}} \subseteq \operatorname{End}_{\mathcal{O}}(\mathcal{L} / M)$ also satisfies Condition[1.1. As in Remark 1.1, the condition that $M$ is isolated and admits a complement may be dropped at the cost of disregarding finitely many (further) prime ideals $\mathfrak{p}$ of $\mathcal{O}$.

In contrast to Assumption 1.1. Condition 1.1 does delineate an interesting property. In Section 4 we discuss, along with several examples and applications of Theorem 1.2 in the context of ideal zeta functions of nilpotent Lie lattices, a number of known examples of such lattices whose generic local ideal zeta functions do not satisfy the kind of functional equations established by Theorem 1.2. We also comment in Section 4 on connections between Theorem 1.2 and related work by du Sautoy and Woodward. To our knowledge, in all cases of ideal zeta functions of nilpotent Lie lattices which are known to satisfy generic local functional equations, Condition 1.1 is satisfied, supporting the speculation that it may actually be necessary for such functional equations. An analogy with Igusa's local zeta function, however, suggests a caveat; cf. Section 3.1.

Indeed, Theorem 1.2 may be viewed as an analogue of the functional equation for the generic Igusa local zeta functions associated to a homogeneous polynomial over $\mathcal{O}$; 9 . In the light of this analogy, Condition 1.1 may be viewed as a "homogeneity condition" for nilpotent algebras of endomorphisms. For a further discussion of the connection with Igusa's local zeta function, necessary vs. sufficient conditions for local functional equations for submodule zeta functions, and potential interpretations of the left-hand side of (1.3), see Section 3 .

The proof of Theorem 1.2 is given in Section 2.1 .

1.2. Applications I: normal zeta functions of finitely generated nilpotent groups. Results such as Theorem 1.2 about ideal zeta functions of nilpotent Lie lattices have corollaries pertaining to normal subgroup zeta functions of finitely generated nilpotent groups, enumerating the groups' normal subgroups of finite index. Indeed, by the Mal'cev correspondence, for every finitely generated torsion-free nilpotent group $G$ there exists a nilpotent Lie ring (viz. $\mathbb{Z}$-Lie lattice) $\mathcal{L}$ such that, for almost all primes $p$, the local ideal zeta function $\zeta_{\mathcal{L}\left(\mathbb{Z}_{p}\right)}(s)$ coincides with the local normal subgroup zeta function

$$
\zeta_{G, p}^{\triangleleft}(s)=\sum_{H \triangleleft p}|G: H|^{-s}
$$

of $G$ at $p$, enumerating normal subgroups of $G$ of $p$-power index in $G$; see [16, Theorem 4.1]. Moreover, every nilpotent Lie ring arises in this way.

Applications of Theorem 1.2 to ideal zeta functions of nilpotent Lie lattices are discussed in Section 4. Via the Mal'cev correspondence, all of them have analogues for normal subgroup zeta functions of finitely generated nilpotent groups. We only spell out the following corollary of Theorem 4.4 on free nilpotent Lie lattices. Let $c, d \in \mathbb{N}$ and $F_{c, d}$ be the free class- $c$-nilpotent group on $d$ generators. For $i \in\{0,1, \ldots, c\}$, set

$$
N_{i}=\sum_{1 \leq j \leq c-i} \frac{1}{j} \sum_{k \mid j} \mu(k) d^{j / k},
$$


where $\mu$ is the Möbius function. This well-known "Witt formula" gives the Hirsch lengths of the quotients of $F_{c, d}$ by the terms of the upper (or, equivalently, lower) central series. The numbers $N_{i}$ are also equal to the $\mathbb{Z}$-ranks of the quotients of the free class- $c$-nilpotent Lie ring on $d$ generators $\mathfrak{f}_{c, d}$ by the terms of the upper central series; cf. Section 4.2. Note that $\mathfrak{f}_{c, d}$ is the nilpotent Lie ring associated to the nilpotent group $F_{c, d}$ by the Mal'cev correspondence. Our Theorem 4.4 on the generic local ideal zeta functions of these Lie rings has the following consequence.

Corollary 1.9. For almost all primes $p$, the following functional equation holds:

$$
\left.\zeta_{F_{c, d}, p}^{\triangleleft}(s)\right|_{p \rightarrow p^{-1}}=(-1)^{N_{0}} p^{\left(\begin{array}{c}
N_{0} \\
2
\end{array}\right)-s\left(\sum_{i=0}^{c-1} N_{i}\right)} \zeta_{F_{c, d}, p}^{\triangleleft}(s) .
$$

For $c>2$ we have, in general, no means of quantifying the respective finite sets of primes for which (1.8) fails to hold. For $c \leq 2$, they are empty: cf. (1.5) for $c=1$ and [34, Theorem 3] for $c=2$.

1.3. Applications II: degree in $q^{-f s}$ and behaviour at $s=-\infty$. Let $(\mathcal{L}, \mathcal{E})$ be as in Section 1.1 with $\mathcal{E}$ nilpotent, not necessarily satisfying Condition 1.1. The following definition is analogous to the concept of uniform representability of families of local zeta functions developed in [21, Section 2.3]; see also [13, § 1.2.4].

Definition 1.10. We call the pair $(\mathcal{L}, \mathcal{E})$ almost uniform if there exists a rational function $W \in \mathbb{Q}(X, Y)$ such that, for almost all prime ideals $\mathfrak{p}$ of $\mathcal{O}$ and all finite extensions $\mathfrak{O}$ of $\mathcal{O}_{\mathfrak{p}}$, with residue field cardinality $q^{f}$, say, $\zeta_{\mathcal{E}(\mathfrak{O}) \curvearrowright \mathcal{L}(\mathfrak{O})}(s)=W\left(q^{f}, q^{-f s}\right)$. By abuse of notation we also call $\zeta_{\mathcal{E} \curvearrowright \mathcal{L}}(s)$ almost uniform in this case.

Recall that, for general reasons, for all $\mathfrak{p}$ and all $\mathfrak{O}$, the local submodule zeta function $\zeta_{\mathcal{E}(\mathfrak{O}) \curvearrowright \mathcal{L}(\mathfrak{O})}(s)$ is a rational function in $q^{-f s}$. The degree of a rational function $W=P / Q \in$ $\mathbb{Q}(Z)$ is $\operatorname{deg}_{Z} W=\operatorname{deg}_{Z} P-\operatorname{deg}_{Z} Q$.

Conjecture 1.11. For almost all prime ideals $\mathfrak{p}$ of $\mathcal{O}$ and all finite extensions $\mathfrak{O}$ of $\mathcal{O}_{\mathfrak{p}}$, with residue field cardinality $q^{f}$, say,

$$
\begin{aligned}
\operatorname{deg}_{q^{-f s}}\left(\zeta_{\mathcal{E}(\mathfrak{O}) \curvearrowright \mathcal{L}(\mathfrak{O})}(s)\right) & =-\sum_{i=0}^{c-1} N_{i}, \\
\lim _{s \rightarrow-\infty}\left(q^{-f s \sum_{i=0}^{c-1} N_{i}} \zeta_{\mathcal{E}(\mathfrak{O}) \curvearrowright \mathcal{L}(\mathfrak{O})}(s)\right) & =(-1)^{n} q^{-f\left(\begin{array}{c}
n \\
2
\end{array}\right) .}
\end{aligned}
$$

If $(\mathcal{L}, \mathcal{E})$ is almost uniform, say $\zeta_{\mathcal{E}(\mathfrak{O}) \curvearrowright \mathcal{L}(\mathfrak{O})}(s)=W\left(q^{f}, q^{-f s}\right)$ for almost all $\mathfrak{p}$ and all $\mathfrak{O}$ for some $W \in \mathbb{Q}(X, Y)$, then $\operatorname{deg}_{X} W=-\left(\begin{array}{c}n \\ 2\end{array}\right)$.

Informally speaking, equation (1.10) pins down the quotient of the leading coefficients of the polynomials in $q^{-f s}$ giving numerator and denominator of the rational function $\zeta_{\mathcal{E}(\mathfrak{O}) \curvearrowright \mathcal{L}(\mathfrak{O})}(s) \in \mathbb{Q}\left(q^{-f s}\right)$. The functional equation (1.3) established in Theorem 1.2 allows us to confirm this conjecture in the case that Condition 1.1 is satisfied. The following is proven in Section 2.2.

Corollary 1.12. Conjecture 1.11 holds if $(\mathcal{L}, \mathcal{E})$ satisfies Condition 1.1 .

Further evidence for Conjecture 1.11 is provided by the numerous examples of ideal zeta functions of nilpotent Lie rings in [13] which do not satisfy generic local functional equations; cf. Section 4 .

Recall that if the degree $\operatorname{deg}_{Y} W$ of a rational generating function $W=P / Q \in \mathbb{Q}(Y)$ is nonpositive, then $\operatorname{deg}_{Y} Q$ is the length of a shortest linear recurrence relation satisfied 
by the coefficients of $W$ when expanded as a power series in $Y$; cf. [31, Theorem 4.1.1]. Equation (1.9) thus yields a lower bound on the length of such a linear recurrence relation. Determining this shortest length precisely seems to be a challenging problem.

Remark 1.13. Our conjecture (1.10) on the behaviour of $\zeta_{\mathcal{E}(\mathfrak{O}) \curvearrowright \mathcal{L}(\mathfrak{O})}(s)$ at $s=-\infty$ may be compared with the conjectural behaviour at $s=0$. In [23, Conjecture IV ( $\mathfrak{P}$-adic form)] Rossmann conjectures that, for all (!) $\mathfrak{p}$ and all $\mathfrak{O}$,

$$
\left.\left(1-q^{-f s}\right) \zeta_{\mathcal{E}(\mathfrak{O}) \curvearrowright \mathcal{L}(\mathfrak{O})}(s)\right|_{s=0}=\frac{1}{\left(1-q^{f}\right)\left(1-q^{2 f}\right) \ldots\left(1-q^{(n-1) f}\right)}
$$

cf. also [23, Section 8.3].

1.4. Context and related work - zeta functions of groups, rings, and algebras. Our proof of Theorem 1.2, presented in Section 2.1. proceeds by adapting the p-adic machinery developed in [35]. There, this technique is applied to establish generic local functional equations for a range of zeta functions of groups and rings. The most general of these applications is to subring zeta functions of arbitrary rings of finite additive rank, i.e. finitely generated abelian groups with some bi-additive multiplicative structure; see [35, Theorem A]. Via the Mal'cev correspondence, this translates into results for the generic Euler factors of the subgroup zeta functions of finitely generated nilpotent groups, i.e. Dirichlet generating series enumerating all finite index subgroups of such a group; see [35, Corollary 1.1]. In [35, Theorem C] we prove functional equations for generic local ideal zeta functions of nilpotent Lie rings of class 2 (or, equivalently, generic local normal zeta functions of finitely generated nilpotent groups of class 2). Theorem 1.2 generalizes this result; cf. Remark 1.8 .

Numerous examples of ideal zeta functions of nilpotent Lie rings have been computed by various people; see, for instance, [13, Section 2] for a substantial list. For a large number of submodule zeta functions associated to nilpotent algebras of endomorphisms see [24] and the database in the computer algebra package [22. The paper [25] gives an explicit formula for the zeta function enumerating submodules invariant under a single nilpotent endomorphism; cf. Section 5.1] for details.

A variant of the subgroup zeta function of a finitely generated nilpotent group $G$ is its pro-isomorphic zeta function $\zeta_{G}^{\wedge}(s)$, enumerating the finite index subgroups of $G$ whose profinite completions are isomorphic to the profinite completion of $G$. These zeta functions, too, enjoy an Euler product $\zeta_{G}^{\wedge}(s)=\prod_{p \text { prime }} \zeta_{G, p}^{\wedge}(s)$ whose factors are rational functions in $p^{-s}$. There are numerous examples of groups whose local pro-isomorphic zeta functions satisfy functional equations akin to (1.3) (see [12, 2] and [5, 4]), but also an example showing that this symmetry phenomenon is not universal for pro-isomorphic zeta functions (see [3]). Formulating necessary and sufficient criteria for generic local functional equations in this context remains an interesting challenge.

In [37, Woodward computed the ideal zeta functions of the full upper triangular $n \times n$ matrices over $\mathbb{Z}$, as well as a number of combinatorially defined quotients of these algebras. He gives sufficient criteria for local functional equations, as well as some examples suggesting that these criteria might be necessary.

Local functional equations akin to (1.3) are also ubiquitous in the theory of representation zeta functions associated to arithmetic (and related pro- $p$ ) groups; see, for instance, [1, 32].

1.5. Notation. We write $\mathbb{N}$ for the natural numbers $\{1,2, \ldots\}$. Given a subset $I \subseteq \mathbb{N}$, we write $I_{0}$ for $I \cup\{0\}$. Given $m, n \in \mathbb{N}_{0}$, we set $[m]=\{1,2, \ldots, m\}$ and $\left.] m, n\right]=$ $\{m+1, \ldots, n\}$. We write $\operatorname{diag}\left(\lambda_{1}^{\left(e_{1}\right)}, \ldots, \lambda_{m}^{\left(e_{m}\right)}\right)$ for the diagonal matrix composed of the 
matrices $\lambda_{1} \operatorname{Id}_{e_{1}}, \ldots, \lambda_{m} \operatorname{Id}_{e_{m}}$. Given matrices $A_{1}, \ldots, A_{n}$ with the same number of rows, we denote by $\left(A_{1}|\ldots| A_{n}\right)$ the matrix obtained by juxtaposition.

Throughout, $p$ is a rational prime, $\mathcal{O}$ the ring of integers of a number field, and $\mathfrak{p}$ a nonzero prime ideal of $\mathcal{O}$. We write $\mathfrak{o}$ to denote a compact discrete valuation ring of arbitrary characteristic, with maximal ideal $\mathfrak{p}$, residue field $\mathfrak{o} / \mathfrak{p}$ of cardinality $q$ and characteristic $p$, and $\mathfrak{p}$-adic valuation $v$ (normalized so that every uniformizer of $\mathfrak{o}$ has valuation 1). If $\mathfrak{o}$ is of characteristic zero, then it is of the form $\mathcal{O}_{\mathfrak{p}}$, the completion of $\mathcal{O}$ at a non-zero prime ideal $\mathfrak{p}$. Note that these rings are exactly the finite extensions of the $p$-adic integers $\mathbb{Z}_{p}$. In this case we denote by $\mathfrak{O}$ a finite extension of $\mathfrak{o}$, with maximal ideal $\mathfrak{P}$ and residue field cardinality $|\mathfrak{O} / \mathfrak{P}|=q^{f}$. In other words, $f=f(\mathfrak{O}, \mathfrak{o})$ is the relative degree of inertia. Given a matrix $A=\left(A_{i, j}\right) \in \operatorname{Mat}_{m, n}(\mathfrak{o})$ we write $v(A)=\min \left\{v\left(A_{i, j}\right) \mid\right.$ $i \in[m], j \in[n]\}$ for the minimal valuation of its entries.

The "Kronecker delta" $\delta_{P}$ associated to a property $P$ is equal to 1 if $P$ holds and equal to 0 otherwise.

\section{Proofs of Theorem 1.2 and Corollary 1.12}

\subsection{Proof of Theorem 1.2,}

2.1.1. Overview of the proof. Let $\mathfrak{p}$ be a nonzero prime ideal of $\mathcal{O}$. We write $\mathfrak{o}=\mathcal{O}_{\mathfrak{p}}$ and $K_{\mathfrak{p}}$ for the field of fractions of $\mathfrak{o}$. We are looking to establish the functional equation (1.3) for almost all zeta functions $\zeta_{\mathcal{E}(\mathfrak{o}) \curvearrowright \mathcal{L}(\mathfrak{o})}(s)$, enumerating the $\mathfrak{o}$-submodules $\Lambda$ of finite index in $\mathcal{L}(\mathfrak{o}) \cong \mathfrak{o}^{n}$ which are also $\mathcal{E}(\mathfrak{o})$-submodules, written $\Lambda \leq_{\mathcal{E}(\mathfrak{o})} \mathcal{L}(\mathfrak{o})$. Clearly the latter property is really a property of the integral members of the homothety class $[\Lambda]=\{x \Lambda \mid$ $\left.x \in K_{\mathfrak{p}}^{*}\right\}$ of $\Lambda$ in $K_{\mathfrak{p}}^{n}$ : either all elements of $[\Lambda]$ are $\mathcal{E}(\mathfrak{o})$-submodules, or none are. We write $[\Lambda] \leq \mathcal{E}(\mathfrak{o}) \mathcal{L}(\mathfrak{o})$ in the former case and set

$$
\operatorname{SubMod}_{\mathcal{E}(\mathfrak{o})}=\left\{[\Lambda] \mid \Lambda \mathfrak{o} \text {-lattice in } K_{\mathfrak{p}}^{n},[\Lambda] \leq_{\mathcal{E}(\mathfrak{o})} \mathcal{L}(\mathfrak{o})\right\} .
$$

Evidently, every homothety class $[\Lambda]$ of $\mathfrak{o}$-lattices in $K_{\mathfrak{p}}^{n}$ contains a unique element $\Lambda_{\max }$ which is contained in $\mathcal{L}(\mathfrak{o})$ and maximal with respect to this property. As the intersection of $[\Lambda]$ with the set of all full sublattices of $\mathcal{L}(\mathfrak{o})$ equals $\left\{\mathfrak{p}^{m} \Lambda_{\max } \mid m \in \mathbb{N}_{0}\right\}$ it thus suffices - in principle - to describe the elements of $\operatorname{SubMod}_{\mathcal{E}(\mathfrak{o})}$ and to keep track of their maximal integral members' indices in $\mathcal{L}(\mathfrak{o})$. Indeed,

$$
\zeta_{\mathcal{E}(\mathfrak{o}) \curvearrowright \mathcal{L}(\mathfrak{o})}(s)=\frac{1}{1-q^{-n s}} \sum_{[\Lambda] \in \operatorname{SubMod}_{\mathcal{E}(\mathfrak{o})}}\left|\mathcal{L}(\mathfrak{o}): \Lambda_{\max }\right|^{-s} .
$$

Geometrically, one may view the set of homothety classes of o-lattices in $K_{\mathfrak{p}}$ as the set of vertices $\mathcal{V}_{n}$ of the affine Bruhat-Tits building $\Delta\left(\mathrm{SL}_{n}\left(K_{\mathfrak{p}}\right)\right)$; see, for instance, [15, Chapter 19]. Keeping track of the indices $\left|\mathcal{L}(\mathfrak{o}): \Lambda_{\max }\right|$ is easy (cf. (2.5)), so the problem of computing the right-hand side of (2.1) is to identify $\operatorname{SubMod}_{\mathcal{E}(\mathfrak{o})}$ as a subset of $\mathcal{V}_{n}$. We give an informal overview of our way to address this challenge, deferring details for the time being.

Firstly, we define an equivalence relation $\sim$ on $\mathcal{V}_{n}$ (viz. $\delta$-equivalence; cf. Definition 2.5) with the property that - provided $\mathcal{E} \neq\{0\}$, as we may assume without loss of generality each $\sim$-class $\mathcal{C}$ is a totally ordered set naturally isomorphic to $(\mathbb{Z}, \leq)$. Moreover, the sets $\mathcal{C}_{\geq 0}:=\mathcal{C} \cap \operatorname{SubMod}_{\mathcal{E}(\mathfrak{o})}$ correspond, under these isomorphisms, to $\left(\mathbb{N}_{0}, \leq\right)$. Setting

$$
\Xi_{\mathcal{C}_{\geq 0}}(s)=\sum_{[\Lambda] \in \mathcal{C}_{\geq 0}}\left|\mathcal{L}(\mathfrak{o}): \Lambda_{\max }\right|^{-s}
$$


we thus obtain

$$
\zeta_{\mathcal{E}(\mathfrak{o}) \curvearrowright \mathcal{L}(\mathfrak{o})}(s)=\frac{1}{1-q^{-n s}} \sum_{\mathcal{C} \in \mathcal{V}_{n} / \sim} \Xi_{\mathcal{C}_{\geq 0}}(s) .
$$

Rather than to analyze the functions $\Xi_{\mathcal{C}_{>0}}(s)$ directly, we extend the sums (2.2) defining them to the whole of $\mathcal{C}$ in a way that allows us to recover the original sum algebraically, uniformly over all $\mathcal{C}$. The motivation to consider these extensions in the first place is that they give rise to a Dirichlet generating series $A^{\triangleleft}(s)$ satisfying a functional equation of the desired type; cf. (2.9).

To this end we introduce, secondly, judiciously chosen functions $\widetilde{m}_{1}, m_{2}: \mathcal{V}_{n} \rightarrow \mathbb{N}_{0}$ with the properties

$$
[\Lambda] \in \operatorname{SubMod}_{\mathcal{E}(\mathfrak{o})} \Leftrightarrow \widetilde{m}_{1}([\Lambda])=0, \quad[\Lambda] \in \operatorname{SubMod}_{\mathcal{E}(\mathfrak{o})} \Rightarrow m_{2}([\Lambda])=0 .
$$

The function $\widetilde{m}_{1}$ may be thought of as measuring a kind of "distance" to $\operatorname{SubMod}_{\mathcal{E}(\mathfrak{o})}$. We set, for each $\mathcal{C} \in \mathcal{V}_{n} / \sim$,

$$
\Xi_{\mathcal{C}}(s)=\sum_{[\Lambda] \in \mathcal{C}}\left|\mathcal{L}(\mathfrak{o}): \Lambda_{\max }\right|^{-s} q^{-s\left((c-1) \tilde{m}_{1}([\Lambda])-m_{2}([\Lambda])\right)},
$$

naturally extending the sum (2.2) defining $\Xi_{\mathcal{C} \geq 0}(s)$. Our choices of $\widetilde{m}_{1}$ and $m_{2}$ will ensure that - up to a power of $q^{-s}$ depending on $\mathcal{C}$ - the function $\Xi_{\mathcal{C}}(s)$ is the sum of two geometric progressions, covering respectively the "nonnegative" part $\mathcal{C}_{\geq 0}$ and the "negative" part $\mathcal{C}_{<0}:=\mathcal{C} \backslash \mathcal{C}_{\geq 0}$ of $\mathcal{C}$, which only depend on the data $\left(N_{i}\right)_{i=0}^{c-1}$ but, crucially, not on $\mathcal{C}$; cf. Corollary 2.7 and Lemma 2.10. This entails that, with

$$
\Xi(s):=\frac{1-q^{-s \sum_{i=1}^{c-1}\left(n-N_{i}\right)}}{1-q^{-s(c-1) n}},
$$

we have $\Xi_{\mathcal{C}_{\geq 0}}(s)=\Xi_{\mathcal{C}}(s) \Xi(s)$ for all $\sim$-classes $\mathcal{C}$. As indicated above, the point of these constructions is that the functions $\widetilde{m}_{1}$ and $m_{2}$ are defined in such a way that - at least for almost all $\mathfrak{p}$ - the function

$$
A^{\triangleleft}(s):=\sum_{\mathcal{C} \in \mathcal{V}_{n} / \sim} \Xi_{\mathcal{C}}(s)=\sum_{[\Lambda] \in \mathcal{V}_{n}}\left|\mathcal{L}(\mathfrak{o}): \Lambda_{\max }\right|^{-s} q^{-s\left((c-1) \tilde{m}_{1}([\Lambda])-m_{2}([\Lambda])\right)}
$$

may be expressed in terms of $\mathfrak{p}$-adic integrals which fit the templates provided by [35. In particular, it satisfies, for almost all $\mathfrak{p}$, the functional equation (2.9). Moreover,

$$
\begin{aligned}
\zeta_{\mathcal{E}(\mathfrak{o}) \curvearrowright \mathcal{L}(\mathfrak{o})}(s)=\frac{1}{1-q^{-n s}} \sum_{\mathcal{C} \in \mathcal{V}_{n} / \sim} \Xi_{\mathcal{C}_{\geq 0}}(s)= & \\
& \frac{1}{1-q^{-n s}} \Xi(s) \sum_{\mathcal{C} \in \mathcal{V}_{n} / \sim} \Xi_{\mathcal{C}}(s)=\frac{1}{1-q^{-n s}} \Xi(s) A^{\triangleleft}(s) .
\end{aligned}
$$

The factor $\frac{1}{1-q^{-n s}} \Xi(s)$ trivially satisfies a functional equation of the required type; see (2.10). Together with (2.9), this will prove Theorem 1.2

2.1.2. Cocentral bases. For $i \in[c]_{0}$ we write $n_{i}=\operatorname{rk}_{\mathcal{O}}\left(\mathcal{L}_{i}\right)$, so that $N_{i}=\operatorname{rk}_{\mathcal{O}}\left(\mathcal{L} / Z_{i}\right)=$ $\sum_{j \leq c-i} n_{j}$. An $\mathcal{O}$-basis $\mathbf{e}=\left(e_{1}, \ldots, e_{n}\right)$ of $\mathcal{L}$ is called cocentral if

$$
Z_{i}=\left\langle e_{N_{i}+1}, \ldots, e_{n}\right\rangle_{\mathcal{O}}
$$

for all $i \in[c]$. (This terminology extends the one introduced in [13, Definition 4.37].) By Assumption 1.1, cocentral bases clearly exist. Condition 1.1 is equivalent to the following condition. 
Condition 2.1. There exist generators $c_{1}, \ldots, c_{d}$ of $\mathcal{E}$ and a cocentral $\mathcal{O}$-basis e of $\mathcal{L}$ such that, for all $k \in[d]$, the matrix $C_{k}$ representing $c_{k}$ with respect to e (acting from the right on row vectors) has the form

$$
C_{k}=\left(C_{k}^{(i j)}\right)_{i, j \in[c]} \in \operatorname{Mat}_{n}(\mathcal{O})
$$

for blocks $C_{k}^{(i j)} \in \operatorname{Mat}_{n_{i}, n_{j}}(\mathcal{O})$ which are zero unless $j=i+1$.

2.1.3. Lattices, matrices, and the submodule condition. Let e be a cocentral $\mathcal{O}$-basis of $\mathcal{L}$ as in Condition 2.1. It yields an $\mathfrak{o}$-basis of $\mathcal{L}(\mathfrak{o})$ which we also denote by $\mathbf{e}$ and which allows us to identify the $\mathfrak{o}$-module $\mathcal{L}(\mathfrak{o})$ with $\mathfrak{o}^{n}$ and $\mathcal{E}(\mathfrak{o})$ with a nilpotent subalgebra of $\operatorname{Mat}_{n}(\mathfrak{o})$, generated by matrices $C_{1}, \ldots, C_{d}$ representing the $\mathfrak{o}$-linear operators $c_{1}, \ldots, c_{d}$. Set $\Gamma=\operatorname{GL}_{n}(\mathfrak{o})$. A full $\mathfrak{o}$-sublattice $\Lambda$ of $\mathcal{L}(\mathfrak{o})$ may be identified with a coset $\Gamma M$ for a matrix $M \in \mathrm{GL}_{n}\left(K_{\mathfrak{p}}\right) \cap \operatorname{Mat}_{n}(\mathfrak{o})$, whose rows encode the coordinates with respect to e of a set of generators of $\Lambda$. Let $\pi \in \mathfrak{p}$ be a uniformizer of $\mathfrak{o}$. By the elementary divisor theorem there exist $I=\left\{i_{1}, \ldots, i_{l}\right\}<\subseteq[n-1], r_{n} \in \mathbb{N}_{0}$, and $\mathbf{r}_{I}=\left(r_{i_{1}}, \ldots, r_{i_{l}}\right) \in \mathbb{N}^{l}$, all uniquely determined by $\Lambda$, and $\alpha \in \Gamma$ such that $M=D \alpha^{-1}$, where

$$
D=\pi^{r_{n}} \operatorname{diag}\left(\left(\pi^{\sum_{\iota \in I} r_{\iota}}\right)^{\left(i_{1}\right)},\left(\pi^{\sum_{\iota \in I \backslash\left\{i_{1}\right\}} r_{\iota}}\right)^{\left(i_{2}-i_{1}\right)}, \ldots,\left(\pi^{r_{i} l}\right)^{\left(i_{l}-i_{l-1}\right)}, 1^{\left(n-i_{l}\right)}\right) \in \operatorname{Mat}_{n}(\mathfrak{o}) .
$$

We write $\nu([\Lambda])=\left(I, \mathbf{r}_{I}\right)$. Note that $r_{n}=v(M)$ in the notation introduced in Section 1.5.

The matrix $\alpha \in \Gamma$ is only unique up to right multiplication by an element of a finite index subgroup $\Gamma_{I, \mathbf{r}}$ of $\Gamma$; see [35, Section 3.1] for details. Obviously,

$$
|\mathcal{L}(\mathfrak{o}): \Lambda|=q^{v(\operatorname{det} D)}=q^{\sum_{\iota \in I \cup\{n\}}{ }^{\iota r_{\iota}}} .
$$

We call $\Lambda$ maximal if $r_{n}=0$ and denote by $\Lambda_{\max }$ the unique maximal integral element of $[\Lambda]$. In the sequel we will often - and sometimes without explicit mentioning - toggle between lattices $\Lambda$ and representing matrices $M$, extending notation for lattices to matrices representing them. We write, for instance, $[M]$ for the homothety class $[\Lambda]$ of the lattice $\Lambda$ determined by $\Gamma M$ and $M \leq_{\mathcal{E}(\mathfrak{o})} \mathcal{L}(\mathfrak{o})$ if $\Lambda \leq_{\mathcal{E}(\mathfrak{o})} \mathcal{L}(\mathfrak{o})$. We also write, given matrices $A$ and $B$ over $\mathfrak{o}$ with the same number of columns, $A \leq B$ if each row of $A$ is contained in the o-row span of $B$.

Define the diagonal matrix

$$
\delta:=\operatorname{diag}\left(\left(\pi^{c-1}\right)^{\left(n_{1}\right)}, \ldots,(\pi)^{\left(n_{c-1}\right)}, 1^{\left(n_{c}\right)}\right) \in \operatorname{Mat}_{n}(\mathfrak{o}) .
$$

We remark that, up to a scalar factor, $\delta$ represents a map closely related to the map $\pi_{\mathcal{B}}$ in [13, Definition 4.40]. Note that $\operatorname{det} \delta=\pi^{\sum_{i=1}^{c-1} N_{i}}$. The following is a trivial consequence of Condition 2.1; for our purposes it is key nevertheless.

Lemma 2.1. If $c>1$, then $\delta C_{k} \delta^{-1}=\pi C_{k}$ for all $k \in[d]$.

For $i \in[n]$ and $k \in[d]$, write $\left(e_{i}\right) c_{k}=\sum_{l=1}^{n} \lambda_{i, k}^{l} e_{l}$ for $\lambda_{i, k}^{l} \in \mathcal{O}$. Then $C_{k}$ satisfies $\left(C_{k}\right)_{r, s}=\lambda_{r, k}^{s}$ for $r, s \in[n]$. Let $\mathbf{Y}=\left(Y_{1}, \ldots, Y_{n}\right)$ be independent variables and set

$$
\mathcal{R}(\mathbf{Y})=\left(\sum_{l=1}^{n} \lambda_{i, k}^{l} Y_{l}\right)_{i, k} \in \operatorname{Mat}_{n \times d}(\mathcal{O}[\mathbf{Y}]) .
$$

Note that $\mathcal{R}=0 \Leftrightarrow \mathcal{E}=0 \Leftrightarrow c=1$. In this case, Theorem 1.2 holds (cf. Example 1.4), so we may assume that $c>1$. We write $\alpha[i]$ for the $i$-th column of a matrix $\alpha \in \Gamma$, so that $\mathcal{R}(\alpha[i]) \in \operatorname{Mat}_{n \times d}(\mathfrak{o})$. The following lemma is verified by a trivial computation. 
Lemma 2.2. For all $\alpha \in \Gamma$ and $\Delta \in \operatorname{Mat}_{n}(\mathfrak{o})$, and $D$ as in (2.4),

$$
\left(\forall k \in[d]: \Delta C_{k} \alpha \leq D\right) \Leftrightarrow\left(\forall i \in[n]: \Delta \mathcal{R}(\alpha[i]) \equiv 0 \bmod D_{i, i}\right) .
$$

Proposition 2.3. Given $M \in \mathrm{GL}_{n}\left(K_{\mathfrak{p}}\right) \cap \operatorname{Mat}_{n}(\mathfrak{o})$, there exists a unique $\widetilde{m}_{1}=\widetilde{m}_{1}(M) \in$ $\mathbb{N}_{0}$ such that, for all $m \in \mathbb{N}_{0}$,

$$
M \delta^{m} \leq_{\mathcal{E}(\mathfrak{o})} \mathcal{L}(\mathfrak{o}) \text { if and only } m \geq \widetilde{m}_{1} .
$$

In particular, $M \leq_{\mathcal{E}(\mathfrak{o})} \mathcal{L}(\mathfrak{o})$ if and only if $\widetilde{m}_{1}=0$. Moreover, $\widetilde{m}_{1} \leq \sum_{\iota \in I} r_{\iota}$.

Proof. Write $M=D \alpha^{-1}$ as above. Without loss we may assume $r_{n}=0$. Using Lemmas 2.1 and 2.2 and the fact that $D_{i, i}=\pi^{\sum_{i \leq \iota \in I} r_{\iota}}$ for $i \in[n]$, we obtain

$$
\begin{aligned}
M \delta^{m} \leq \mathcal{E}(\mathfrak{o}) \mathcal{L}(\mathfrak{o}) & \Leftrightarrow \forall k \in[d]: M \delta^{m} C_{k} \leq M \delta^{m} \\
& \Leftrightarrow \forall k \in[d]: D \alpha^{-1} \delta^{m} C_{k} \delta^{-m} \alpha \leq D \\
& \Leftrightarrow \forall k \in[d]: \pi^{m} D \alpha^{-1} C_{k} \alpha \leq D \\
\Leftrightarrow & \forall i \in[n]: \pi^{m} D \alpha^{-1} \mathcal{R}(\alpha[i]) \equiv 0 \bmod D_{i, i} \\
\Leftrightarrow & \forall i \in[n]: \pi^{m} D \alpha^{-1} \mathcal{R}(\alpha[i]) \pi^{\sum_{i>\iota \in I} r_{\iota}} \equiv 0 \bmod \pi^{\sum_{\iota \in I} r_{\iota}} \\
\Leftrightarrow & \pi^{m} D \alpha^{-1}(\mathcal{R}(\alpha[1])|\cdots| \mathcal{R}(\alpha[n])) \cdot \\
& \operatorname{diag}\left(1^{\left(d i_{1}\right)},\left(\pi^{r_{i_{1}}}\right)^{\left(d\left(i_{2}-i_{1}\right)\right)}, \ldots,\left(\pi^{\sum_{\iota \in I} r_{\iota}}\right)^{\left(d\left(n-i_{l}\right)\right)}\right) \equiv 0 \bmod \pi^{\sum_{\iota \in I} r_{\iota}} .
\end{aligned}
$$

In the last congruence, we may replace $\alpha^{-1}$ by the adjunct matrix $\alpha^{\text {adj }}$. Setting, for $i, r \in[n]$,

$$
\begin{aligned}
\mathcal{R}_{(i)}(\alpha) & =\alpha^{\operatorname{adj}} \mathcal{R}(\alpha[i]), \\
v_{i, r}^{(1)}(\alpha) & =\min \left\{v\left(\mathcal{R}_{(\iota)}(\alpha)_{\rho \sigma}\right) \mid \iota \leq i, \rho \geq r, \sigma \in[d]\right\},
\end{aligned}
$$

and

$$
m_{1}(M)=\min \left\{\sum_{\iota \in I} r_{\iota}, \sum_{r \leq \iota \in I} r_{\iota}+\sum_{i>\iota \in I} r_{\iota}+v_{i, r}^{(1)}(\alpha) \mid(i, r) \in[n]^{2}\right\},
$$

we may rephrase the above equivalence as follows:

$$
M \delta^{m} \leq_{\mathcal{E}(\mathfrak{o})} \mathcal{L}(\mathfrak{o}) \Leftrightarrow m \geq \sum_{\iota \in I} r_{\iota}-m_{1}(M)=: \widetilde{m}_{1}(M) .
$$

Definition 2.4. For a lattice $\Lambda$ corresponding to a coset $\Gamma M$, we set $\widetilde{m}_{1}([\Lambda])=\widetilde{m}_{1}(M)$.

Informally, $\widetilde{m}_{1}([\Lambda])$ is a 'distance' in $\mathcal{V}_{n}$ between $[\Lambda]$ and $\operatorname{SubMod}_{\mathcal{E}(\mathfrak{o})}$.

\subsection{4. $\delta$-equivalence. Recall the diagonal matrix $\delta$ defined in (2.6).}

Definition 2.5. Lattice classes $\left[\Lambda_{1}\right],\left[\Lambda_{2}\right] \in \mathcal{V}_{n}$ are called $\delta$-equivalent, written $\left[\Lambda_{1}\right] \sim\left[\Lambda_{2}\right]$, if there exists $m \in \mathbb{Z}$ such that $\left[\Lambda_{1}\right]=\left[\Lambda_{2} \delta^{m}\right]$.

In the sequel, we use the terms lattice class for a homothety class of lattices and $\delta$-class for a $\sim$-equivalence class of lattice classes. Proposition 2.3 asserts that every $\delta$-class of lattice classes intersects $\operatorname{SubMod}_{\mathcal{E}(\mathfrak{o})}$ nontrivially. Its proof also shows that, more precisely, in each $\delta$-class $\mathcal{C}$ there is a unique lattice class $\left[\Lambda_{0}\right]$ such that $\left[\Lambda_{0} \delta^{m}\right] \leq_{\mathcal{E}(\mathfrak{o})} \mathcal{L}(\mathfrak{o})$ if and only 
if $m \in \mathbb{N}_{0}$. We shall say that $\left[\Lambda_{0}\right]$ generates $\mathcal{C}_{\geq 0}$ and write $\Lambda_{0 \text {,max }}$ for the unique maximal element of $\left[\Lambda_{0}\right]$. Setting

$$
\begin{aligned}
& \mathcal{C}_{\geq 0}=\left\{\left[\Lambda_{0} \delta^{m}\right] \mid m \geq 0\right\}=\mathcal{C} \cap \operatorname{SubMod}_{\mathcal{E}(\mathfrak{o})}, \\
& \mathcal{C}_{<0}=\left\{\left[\Lambda_{0} \delta^{m}\right] \mid m<0\right\}=\mathcal{C} \backslash \mathcal{C}_{\geq 0},
\end{aligned}
$$

we obtain a partition $\mathcal{C}=\mathcal{C}_{\geq 0} \cup \mathcal{C}_{<0}$ and (2.3) holds with $\Xi_{\mathcal{C}_{>0}}(s)$ defined as in (2.2).

Note that clearly $v(M) \leq v(M \delta)$ for all $M \in \operatorname{GL}_{n}\left(K_{\mathfrak{p}}\right) \cap \operatorname{Mat}_{n}(\mathfrak{o})$.

Lemma 2.6. For almost all prime ideals $\mathfrak{p}$, the following holds for all $M \in \mathrm{GL}_{n}\left(K_{\mathfrak{p}}\right) \cap$ $\operatorname{Mat}_{n}\left(\mathcal{O}_{\mathfrak{p}}\right)$ : if $M \leq_{\mathcal{E}\left(\mathcal{O}_{\mathfrak{p}}\right)} \mathcal{L}\left(\mathcal{O}_{\mathfrak{p}}\right)$, then $v(M)=v(M \delta)$.

Proof. We proceed by induction on $c$, including the case $c=1$, which we excluded in the previous arguments. Indeed, for this base case the statement holds trivially (and for all $\mathfrak{p}$ ) as $\delta=\operatorname{Id}_{n}$. Assume thus that $c \geq 2$ and that the induction hypothesis holds. Given $\mathfrak{p}$ and $M$, write $\Lambda$ for the lattice defined by $M$ and set $\mathfrak{o}=\mathcal{O}_{\mathfrak{p}}$. Without loss we may assume that $M$ is a block-upper triangular matrix, i.e. composed of blocks $M^{(i j)} \in \operatorname{Mat}_{n_{i}, n_{j}}(\mathfrak{o})$, $i, j \in[c]$, with $M^{(i j)}=0$ unless $j \geq i$.

The claim is that, for almost all $\mathfrak{p}$ and all $M$, the minimal $\mathfrak{p}$-adic valuation of the entries of $M$ is equal to that of its last block column: if $\pi$ divides one of the block matrices $M^{(i c)}$, $i \in[c]$, then it divides the whole matrix $M$. By induction hypothesis (and excluding finitely many $\mathfrak{p}$ ), we may assume that the matrix $M^{\prime}:=\left(M^{(i j)}\right)_{i, j \in[2, c]} \in \operatorname{Mat}_{n-N_{c-1}}(\mathfrak{o})$, defining the lattice $\Lambda \cap Z_{c-1}(\mathfrak{o})$, where $Z_{c-1}(\mathfrak{o})=Z_{c-1} \otimes_{\mathcal{O}} \mathfrak{o}$, has the desired property that if $\pi$ divides the last block column of $M^{\prime}$, viz. one of the block matrices $M^{(i c)}, i \in[2, c]$, then $\pi$ divides the whole matrix $M^{\prime}$. So assume that $\pi$ divides the last block column of $M$ and thus that $M^{(i j)} \equiv 0 \bmod \pi$ for $i \geq 2$ or $j=c$, but that there exists $j \in[c-1]$ such that $v\left(M^{(1 j)}\right)=0$. Thus one of the first $n_{1}$ rows of $M$ is nonzero modulo $\pi$, defining an element $x \in \mathcal{L}(\mathfrak{o}) / Z_{1}(\mathfrak{o})$ which is nonzero modulo $\pi$. But for all but finitely many $\mathfrak{p}$ the reduction modulo $\mathfrak{p}$ of $Z_{1}(\mathfrak{o})$ is the centraliser of $\mathcal{L}(\mathfrak{o} / \mathfrak{p})=\mathcal{L} \otimes_{\mathfrak{o}} \mathfrak{o} / \mathfrak{p}$. This establishes the claim.

Assume from now that $\mathfrak{p}$ satisfies the conclusions of Lemma 2.6.

Corollary 2.7. For every $\mathcal{C} \in \mathcal{V}_{n} / \sim$,

$$
\Xi_{\mathcal{C}_{\geq 0}}(s)=\left|\mathcal{L}(\mathfrak{o}): \Lambda_{0, \max }\right|^{-s} \frac{1}{1-q^{-s \sum_{i=1}^{c-1} N_{i}}} .
$$

Proof. For all $m \in \mathbb{N}_{0}$ we have $\Lambda_{0, \max } \delta^{m}=\left(\Lambda_{0, \max } \delta^{m}\right)_{\max }$ by Lemma 2.6. Hence

$$
\left|\mathcal{L}(\mathfrak{o}): \Lambda_{0, \max } \delta^{m}\right|=\left|\mathcal{L}(\mathfrak{o}): \Lambda_{0, \max }\right| q^{m \sum_{i=1}^{c-1} N_{i}}
$$

and therefore

$$
\begin{aligned}
\Xi_{\mathcal{C}_{\geq 0}}(s)= & \sum_{[\Lambda] \in \mathcal{C}_{\geq 0}}\left|\mathcal{L}(\mathfrak{o}): \Lambda_{\max }\right|^{-s}=\sum_{m=0}^{\infty}\left|\mathcal{L}(\mathfrak{o}): \Lambda_{0, \max } \delta^{m}\right|^{-s}= \\
& \left|\mathcal{L}(\mathfrak{o}): \Lambda_{0, \max }\right|^{-s} \sum_{m=0}^{\infty} q^{-s m \sum_{i=1}^{c-1} N_{i}}=\left|\mathcal{L}(\mathfrak{o}): \Lambda_{0, \max }\right|^{-s} \frac{1}{1-q^{-s \sum_{i=1}^{c-1} N_{i}}}
\end{aligned}
$$

We set $\widetilde{\delta}=\pi^{c-1} \delta^{-1}$ and note that $\operatorname{det} \widetilde{\delta}=\pi^{\sum_{i=1}^{c-1}\left(n-N_{i}\right)}$ and $\mathcal{C}_{<0}=\left\{\left[\Lambda_{0} \widetilde{\delta}^{m}\right] \mid m>0\right\}$. We seek to describe the 'weight function' $w: \mathcal{V}_{n} \rightarrow \mathbb{N}_{0}$ defined by the property that 
$\left.w\right|_{\operatorname{SubMod}_{\mathcal{E}(\mathfrak{o})}}=0$ and, for each $\delta$-class $\mathcal{C}$,

$$
\Xi_{\mathcal{C}_{<0}}(s):=\sum_{[\Lambda] \in \mathcal{C}<0}\left|\mathcal{L}(\mathfrak{o}): \Lambda_{\max }\right|^{-s} q^{-s n w([\Lambda])}=\sum_{m=1}^{\infty}\left|\mathcal{L}(\mathfrak{o}): \Lambda_{0, \max } \widetilde{\delta}^{m}\right|^{-s}
$$

Note that then

$$
\Xi_{\mathcal{C}_{<0}}(s)=\left|\mathcal{L}(\mathfrak{o}): \Lambda_{0, \max }\right|^{-s} \sum_{m=1}^{\infty} q^{-s m \sum_{i=1}^{c-1}\left(n-N_{i}\right)}=\left|\mathcal{L}(\mathfrak{o}): \Lambda_{0, \max }\right|^{-s} \frac{q^{-s \sum_{i=1}^{c-1}\left(n-N_{i}\right)}}{1-q^{-s \sum_{i=1}^{c-1}\left(n-N_{i}\right)}} .
$$

To obtain this analogue of the formula for $\Xi_{\mathcal{C}_{\geq 0}}(s)$ established in Corollary 2.7, we need to take care to define $w$ judiciously: the point here is that, whilst with $\Lambda_{0, \max }$ also $\Lambda_{0, \max } \delta^{m}$ is maximal (cf. Lemma 2.6), the lattice $\Lambda_{0, \max } \widetilde{\delta}^{m}$ is not, in general, as the following example illustrates.

Example 2.8. Let $\mathcal{L}(\mathfrak{o})=\langle x, y, z \mid[x, y]=z,[x, z]=[y, z]=0\rangle_{\mathfrak{o}}$ be the Heisenberg o-Lie lattice. Consider the matrices

$$
M_{1}=\left(\begin{array}{lll}
1 & & \\
& 1 & \\
& & \pi
\end{array}\right), \quad M_{2}=\left(\begin{array}{lll}
1 & & 1 \\
& 1 & 1 \\
& & \pi
\end{array}\right) \in \operatorname{Mat}_{3}(\mathfrak{o})
$$

encoding sublattices of index $q$ in $\mathcal{L}(\mathfrak{o}) \cong \mathfrak{o}^{3}$ with respect to the $\mathfrak{o}$-basis $(x, y, z)$. Clearly neither of them are ideals of $\mathcal{L}(\mathfrak{o})$ (in fact, $\widetilde{m}_{1}\left(M_{1}\right)=\widetilde{m}_{1}\left(M_{2}\right)=1$ ) and their homothety classes are $\delta$-inequivalent. Considering

$$
M_{1} \delta=\left(\begin{array}{ccc}
\pi & & \\
& \pi & \\
& & \pi
\end{array}\right)=\pi \operatorname{Id}_{3}, \quad M_{2} \delta=\left(\begin{array}{lll}
\pi & & 1 \\
& \pi & 1 \\
& & \pi
\end{array}\right),
$$

it is clear that whilst $M_{2} \delta$ is maximal, $M_{1} \delta$ is not. Thus $w\left(\left[M_{1}\right]\right)=0$ but $w\left(\left[M_{2}\right]\right)=1$.

Given $w$ to the requirements of (2.8), it suffices to show that the function

$$
A^{\triangleleft}(s):=\sum_{[\Lambda] \in \mathcal{V}_{n}}\left|\mathcal{L}(\mathfrak{o}): \Lambda_{\max }\right|^{-s} q^{-s n w([\Lambda])}
$$

satisfies the functional equation

$$
\left.A^{\triangleleft}(s)\right|_{q \rightarrow q^{-1}}=(-1)^{n-1} q^{\left(\begin{array}{c}
n \\
2
\end{array}\right)} A^{\triangleleft}(s) .
$$

Indeed, $A^{\triangleleft}(s)$ is, by design of $w$, as a Dirichlet generating series with nonnegative coefficients, divisible by

$$
\underbrace{\frac{1}{1-q^{-s \sum_{i=1}^{c-1} N_{i}}}}_{\text {sum over } \mathcal{C}_{\geq 0}}+\underbrace{\frac{q^{-s \sum_{i=1}^{c-1}\left(n-N_{i}\right)}}{1-q^{-s \sum_{i=1}^{c-1}\left(n-N_{i}\right)}}}_{\text {sum over } \mathcal{C}_{<0}}=\frac{1-q^{-s(c-1) n}}{\left(1-q^{-s \sum_{i=1}^{c-1} N_{i}}\right)\left(1-q^{-s \sum_{i=1}^{c-1}\left(n-N_{i}\right)}\right)} .
$$

We want, however, to isolate the geometric progression $\left(1-q^{-s \sum_{i=1}^{c-1} N_{i}}\right)^{-1}$ taking care of the enumeration over the $\mathcal{C}_{\geq 0}$. Thus, by (2.3),

$$
\begin{aligned}
\zeta_{\mathcal{E}(\mathfrak{o}) \curvearrowright \mathcal{L}(\mathfrak{o})}(s) & =\frac{1}{1-q^{-n s}} \cdot \frac{1}{1-q^{-s \sum_{i=1}^{c-1} N_{i}}} \cdot \frac{\left(1-q^{-s \sum_{i=1}^{c-1} N_{i}}\right)\left(1-q^{-s \sum_{i=1}^{c-1}\left(n-N_{i}\right)}\right)}{1-q^{-s(c-1) n}} A^{\triangleleft}(s) \\
& =\frac{1-q^{-s} \sum_{i=1}^{c-1}\left(n-N_{i}\right)}{\left(1-q^{-n s}\right)\left(1-q^{-s(c-1) n}\right)} A^{\triangleleft}(s)=\frac{1}{1-q^{-n s}} \Xi(s) A^{\triangleleft}(s) .
\end{aligned}
$$


Given (2.9), the functional equation (1.3) follows as, trivially,

$$
\left.\frac{1-q^{-s \sum_{i=1}^{c-1}\left(n-N_{i}\right)}}{\left(1-q^{-n s}\right)\left(1-q^{-s(c-1) n}\right)}\right|_{q \rightarrow q^{-1}}=-q^{-s \sum_{i=0}^{c-1} N_{i}} \frac{1-q^{-s \sum_{i=1}^{c-1}\left(n-N_{i}\right)}}{\left(1-q^{-n s}\right)\left(1-q^{-s(c-1) n}\right)} .
$$

It remains to devise the weight function $w$ to the requirement of (2.8).

Definition 2.9. Given $M \in \operatorname{GL}_{n}\left(K_{\mathfrak{p}}\right) \cap \operatorname{Mat}_{n}(\mathfrak{o})$ corresponding to a maximal lattice $\Lambda$, write $M=\left(M^{(i j)}\right)_{i, j \in[c]}$ as in the proof of Lemma 2.6. Define

$$
m_{2}([\Lambda])=\min \left\{v\left(M^{(i c)}\right) \mid i \in[c]\right\} .
$$

Informally speaking, $m_{2}([\Lambda])$ is the valuation of the last $n_{c}$ columns of a matrix $M$ representing $\Lambda_{\max }$. Recall the "distance" function $\widetilde{m}_{1}: \mathcal{V}_{n} \rightarrow \mathbb{N}_{0}$; see Definition 2.4.

Lemma 2.10. Equation (2.8) holds with $w([\Lambda])=(c-1) \widetilde{m}_{1}([\Lambda])-m_{2}([\Lambda])$.

Proof. Denote by $\left[\Lambda_{0}\right]$ the generator of $\mathcal{C}_{\geq 0}$ and by $\Lambda_{0, \max } \in\left[\Lambda_{0}\right]$ its unique maximal element. It suffices to observe that $v\left(M \delta^{\tilde{m}_{1}([\Lambda])}\right)=m_{2}([\Lambda])$. Hence the matrix $M \delta^{\tilde{m}_{1}([\Lambda])}$ corresponds to the lattice $\pi^{m_{2}([\Lambda])} \Lambda_{0, \max }$, whence

$$
\pi^{-m_{2}([\Lambda])}\left(M \delta^{\widetilde{m}_{1}([\Lambda])}\right) \widetilde{\delta}^{\widetilde{m}_{1}([\Lambda])}=M \pi^{(c-1) \widetilde{m}_{1}([\Lambda])-\widetilde{m}_{2}([\Lambda])}
$$

corresponds to $\Lambda_{0, \max } \widetilde{\delta}^{\tilde{m}_{1}([\Lambda])}$.

For later reference we record another formula for the invariant $m_{2}$. Write $M=D \alpha^{-1}$ as above. Setting, for $r \in[n]$,

$$
\left.\left.v_{r}^{(2)}(\alpha):=\min \left\{v\left(\left(\alpha^{\mathrm{adj}}\right)_{\rho \sigma}\right) \mid \rho \geq r, \sigma \in\right] N_{1}, n\right]\right\},
$$

we obtain

$$
m_{2}([\Lambda])=\min \left\{\sum_{\iota \in I} r_{\iota}, \sum_{r \leq \iota \in I} r_{\iota}+v_{r}^{(2)}(\alpha) \mid r \in[n]\right\} .
$$

To compute $A^{\triangleleft}(s)$ we need, given a lattice class $[\Lambda] \in \mathcal{V}_{n}$ with $\nu([\Lambda])=\left(I, \mathbf{r}_{I}\right)$, to keep track of the quantity

$$
\begin{aligned}
& q^{-s\left(\left(\sum_{\iota \in I} \iota r_{\iota}\right)+n w([\Lambda])\right)}=q^{-s\left(\left(\sum_{\iota \in I} \iota r_{\iota}\right)+n\left((c-1) \tilde{m}_{1}([\Lambda])-m_{2}([\Lambda])\right)\right)}= \\
& q^{-s\left(\left(\sum_{\iota \in I} r_{\iota}(\iota+n(c-1))\right)-n\left((c-1) m_{1}([\Lambda])+m_{2}([\Lambda])\right)\right) .}
\end{aligned}
$$

(Here we used (2.5), Lemma 2.10, and (2.7).) To this end we define, given $I \subseteq[n-1]_{0}$ and $\mathbf{r} \in \mathbb{N}^{|I|}$ as above, for $\mathbf{m}=\left(m_{1}, m_{2}\right) \in \mathbb{N}_{0}^{2}$,

$$
\mathcal{N}_{I, \mathbf{r}, \mathbf{m}}^{\triangleleft}=\left|\left\{[\Lambda] \in \mathcal{V}_{n} \mid \nu([\Lambda])=(I, \mathbf{r}), m_{i}([\Lambda])=m_{i}, i \in\{1,2\}\right\}\right|
$$

and set

$$
A_{I}^{\triangleleft}(s)=\sum_{\mathbf{r} \in \mathbb{N}|I|} q^{-s \sum_{\iota \in I} r_{\iota}(\iota+n(c-1))} \sum_{\mathbf{m}=\left(m_{1}, m_{2}\right) \in \mathbb{N}_{0}^{2}} \mathcal{N}_{I, \mathbf{r}, \mathbf{m}}^{\triangleleft} q^{s n\left((c-1) m_{1}+m_{2}\right)},
$$

so that $A^{\triangleleft}(s)=\sum_{I \subseteq[n-1]} A_{I}^{\triangleleft}(s)$. 
2.1.5. $\mathfrak{p}$-Adic integration. To establish the functional equation (2.9) for $A^{\triangleleft}(s)$ we express each of the functions $A_{I}^{\triangleleft}(s)$ in terms of $\mathfrak{p}$-adic integrals of the form [35, (6)] that satisfy the hypotheses of [35, Theorem 2.3]. To this end we consider multivariate $\mathfrak{p}$-adic integrals $Z_{I}^{\triangleleft}(\mathbf{s})$ (cf. (2.12) $)$ on $\mathfrak{p}^{|I|} \times \Gamma$ whose integrands are defined using carefully chosen polynomial mappings. The latter are designed in such a way that the numbers $\mathcal{N}_{I, \mathbf{r}, \mathbf{m}}^{\triangleleft}$ entering (2.11) may easily be expressed in terms of the (Haar) measures $\mu_{I, \mathbf{r}, \mathbf{m}}^{\triangleleft}$ of the subsets of $\mathfrak{p}^{|I|} \times \Gamma$ on which the integrands are constant; cf. Lemma 2.11. The generating functions $A_{I}^{\triangleleft}(s)$ may be recovered from (suitable normalizations of) the integrals $Z_{I}^{\triangleleft}(\mathbf{s})$ upon a judiciously chosen affine-linear univariate substitution of variables; cf. (2.16).

This type of connection between generating functions and $\mathfrak{p}$-adic integrals has been exploited numerous times before. It links, for instance, Igusa's local zeta function (cf. Section 3.1) with the problem of counting solutions to polynomial congruences modulo powers of $\mathfrak{p}$; cf. [8, Section 1.2]. The formalism set up in [35, Section 2.2] is based on the very same principle.

To return to the problem at hand we define, for $i, r \in[n]$, sets of polynomials

$$
\begin{aligned}
& \mathbf{f}_{i, r}^{(1)}(\mathbf{y})=\left\{\left(\mathcal{R}_{(\iota)}(\mathbf{y})\right)_{\rho \sigma} \mid \iota \leq i, \rho \geq r, \sigma \in[d]\right\}, \\
& \left.\left.\left.\mathbf{f}_{r}^{(2)}(\mathbf{y})=\left\{\left(\mathbf{y}^{\text {adj }}\right)\right)_{\rho \sigma} \mid \rho \geq r, \sigma \in\right] N_{1}, n\right]\right\},
\end{aligned}
$$

and set, for $I \subseteq[n-1]$,

$$
\begin{array}{ll}
\mathbf{g}_{n, I}^{(1)}(\boldsymbol{x}, \mathbf{y})=\left\{\prod_{\iota \in I} x_{\iota}\right\} \cup \bigcup_{(i, r) \in[n]^{2}}\left(\prod_{\iota \in I} x_{\iota}^{\delta_{r \leq \iota}+\delta_{i}>\iota}\right) \mathbf{f}_{i, r}^{(1)}(\mathbf{y}), \\
\mathbf{g}_{n, I}^{(2)}(\boldsymbol{x}, \mathbf{y})=\left\{\prod_{\iota \in I} x_{\iota}\right\} \cup & \bigcup_{r \in[n]}\left(\prod_{\iota \in I} x_{\iota}^{\delta_{r} \leq \iota}\right) \mathbf{f}_{r}^{(2)}(\mathbf{y}),
\end{array}
$$

and, for $\kappa \in[n-1]$,

$$
\mathbf{g}_{\kappa, I}(\boldsymbol{x}, \mathbf{y})=\left\{\prod_{\iota \in I} x_{\iota}^{\delta_{\iota \kappa}}\right\} .
$$

With this data we define the $\mathfrak{p}$-adic integral

$$
\begin{aligned}
Z_{I}^{\triangleleft}(\mathbf{s})= & Z_{I}^{\triangleleft}\left(\left(s_{\iota}\right)_{\iota \in I}, s_{n}^{(1)}, s_{n}^{(2)}\right):= \\
& \int_{\mathfrak{p}^{|I|} \times \Gamma}\left\|\mathbf{g}_{n, I}^{(1)}(\boldsymbol{x}, \mathbf{y})\right\|^{s_{n}^{(1)}}\left\|\mathbf{g}_{n, I}^{(2)}(\boldsymbol{x}, \mathbf{y})\right\|^{s_{n}^{(2)}} \prod_{\kappa \in[n-1]}\left\|g_{\kappa, I}(\boldsymbol{x}, \mathbf{y})\right\|^{s_{\kappa}}\left|\mathrm{d} \boldsymbol{x}_{I} \| \mathrm{d} \mathbf{y}\right| .
\end{aligned}
$$

Here, $\mathbf{s}=\left(s_{1}, \ldots, s_{n-1}, s_{n}^{(1)}, s_{n}^{(2)}\right)$ is a vector of complex variables; note, however, that $s_{\kappa}$ occurs on the right-hand side if and only if $\kappa \in I$. Moreover, $\left|\mathrm{d} \boldsymbol{x}_{I}\right||\mathrm{d} \mathbf{y}|$ denotes the Haar measure, normalized such that the domain of integration has measure $q^{-|I|} \mu(\Gamma)$, where $\mu(\Gamma)=\prod_{i=1}^{n}\left(1-q^{-i}\right)$. By $\|\cdot\|$ we denote the $\mathfrak{p}$-adic (maximum) norm. The integral $Z_{I}^{\triangleleft}(\mathbf{s})$ is, by design, of the form [35, (6)]. Discarding at most finitely many primes, we may assume that the assumptions of [35, Theorem 2.2] are satisfied. This will imply that the normalized integrals

$$
\widetilde{Z_{I}^{\triangleleft}}(\mathbf{s}):=\frac{Z_{I}^{\triangleleft}(\mathbf{s})}{\left(1-q^{-1}\right)^{|I|} \mu(\Gamma)}
$$


(cf. [35, (10)]) satisfy the 'inversion properties' established in [35, Theorem 2.3]. Hence the sum $\widetilde{Z^{\triangleleft}}(\mathbf{s}):=\sum_{I \subseteq[n-1]}\left(\begin{array}{l}n \\ I\end{array}\right)_{q^{-1}} \widetilde{Z_{I}^{\triangleleft}}(\mathbf{s})$ (cf. [35, (16)]) satisfies the functional equation

$$
\left.\widetilde{Z^{\triangleleft}}(\mathbf{s})\right|_{q \rightarrow q^{-1}}=(-1)^{n-1} q^{\left(\begin{array}{c}
n \\
2
\end{array}\right) \widetilde{Z^{\triangleleft}}(\mathbf{s}) ;}
$$

cf. [35, Cor. 2.3]. Here, $\left(\begin{array}{l}n \\ I\end{array}\right)_{X} \in \mathbb{Z}[X]$ denotes the Gaussian multinomial coefficient.

It remains to show that, for each $I \subseteq[n-1]$, the generating function $A_{I}^{\triangleleft}(s)$ is indeed obtainable from the $\mathfrak{p}$-adic integral $Z_{I}^{\triangleleft}(\mathbf{s})$ by a suitable specialization of the variables $\mathbf{s}$. We start by measuring the sets on which the integrand of $Z_{I}^{\triangleleft}(\mathbf{s})$ is constant. More precisely we set, for $\mathbf{m}=\left(m_{1}, m_{2}\right) \in \mathbb{N}_{0}^{2}$ and $\mathbf{r} \in \mathbb{N}^{|I|}$,

$$
\mu_{I, \mathbf{r}, \mathbf{m}}^{\triangleleft}:=\mu\left\{(\boldsymbol{x}, \mathbf{y}) \in \mathfrak{p}^{|I|} \times \Gamma \mid \forall \iota \in I: v\left(x_{\iota}\right)=r_{\iota}, \mathbf{m}(\boldsymbol{x}, \mathbf{y})=\left(m_{1}, m_{2}\right)\right\},
$$

where $\mathbf{m}(\boldsymbol{x}, \mathbf{y})=\left(\mathbf{m}(\boldsymbol{x}, \mathbf{y})_{1}, \mathbf{m}(\boldsymbol{x}, \mathbf{y})_{2}\right)$ and

$$
\begin{aligned}
& \mathbf{m}(\boldsymbol{x}, \mathbf{y})_{1}=\min \left\{\sum_{\iota \in I} r_{\iota}, \sum_{r \leq \iota \in I} v\left(x_{\iota}\right)+\sum_{i>\iota \in I} v\left(x_{\iota}\right)+v_{i, r}^{(1)}(\mathbf{y}) \mid(i, r) \in[n]^{2}\right\}, \\
& \mathbf{m}(\boldsymbol{x}, \mathbf{y})_{2}=\min \left\{\sum_{\iota \in I} r_{\iota}, \sum_{r \leq \iota \in I} v\left(x_{\iota}\right) \quad+v_{r}^{(2)}(\mathbf{y}) \mid r \in[n]\right\} .
\end{aligned}
$$

Then, by design,

$$
\widetilde{Z_{I}^{\triangleleft}}(\mathbf{s})=\frac{1}{\left(1-q^{-1}\right)^{|I|} \mu(\Gamma)} \sum_{\mathbf{r} \in \mathbb{N}|I|} q^{-\sum_{\iota \in I} s_{\iota} r_{\iota}} \sum_{\mathbf{m}=\left(m_{1}, m_{2}\right) \in \mathbb{N}_{0}^{2}} \mu_{I, \mathbf{r}, \mathbf{m}}^{\triangleleft} q^{-s_{n}^{(1)} m_{1}-s_{n}^{(2)} m_{2}} .
$$

The numbers $\mu_{I, \mathbf{r}, \mathbf{m}}^{\triangleleft}$ are closely related to the natural numbers $\mathcal{N}_{I, \mathbf{r}, \mathbf{m}}^{\triangleleft}$ we are looking to control.

\section{Lemma 2.11.}

$$
\mathcal{N}_{I, \mathbf{r}, \mathbf{m}}^{\triangleleft}=\frac{\left(\begin{array}{l}
n \\
I
\end{array}\right)_{q^{-1}}}{\left(1-q^{-1}\right)^{|I|} \mu(\Gamma)} \mu_{I, \mathbf{r}, \mathbf{m}}^{\triangleleft} q^{\sum_{\iota \in I} r_{\iota}(\iota(n-\iota)+1)} .
$$

Proof. Analogous to [35, Lemma 3.1].

Thus, combining (2.11), (2.15), and (2.14), we obtain

$$
\begin{aligned}
A_{I}^{\triangleleft}(s) & =\sum_{\mathbf{r} \in \mathbb{N}|I|} q^{-s \sum_{\iota \in I} r_{\iota}(\iota+n(c-1))} \sum_{\mathbf{m}=\left(m_{1}, m_{2}\right) \in \mathbb{N}_{0}^{2}} \mathcal{N}_{I, \mathbf{r}, \mathbf{m}}^{\triangleleft} q^{s n\left((c-1) m_{1}+m_{2}\right)} \\
& =\frac{\left(\begin{array}{l}
n \\
I
\end{array}\right)_{q^{-1}}}{\left(1-q^{-1}\right)^{|I|} \mu(\Gamma)} \sum_{\mathbf{r} \in \mathbb{N}^{|I|}} q^{-\sum_{\iota \in I} r_{\iota}(s(\iota+n(c-1))-\iota(n-\iota)-1)} \sum_{\mathbf{m} \in \mathbb{N}_{0}^{2}} \mu_{I, \mathbf{r}, \mathbf{m}}^{\triangleleft} q^{s n\left((c-1) m_{1}+m_{2}\right)} \\
(2.16) & =\left(\begin{array}{l}
n \\
I
\end{array}\right)_{q^{-1}} \widetilde{Z_{I}^{\triangleleft}}\left((s(\iota+n(c-1))-\iota(n-\iota)-1)_{\iota \in I},-n s(c-1),-n s\right) .
\end{aligned}
$$

The functional equation (2.9) for $A^{\triangleleft}(s)=\sum_{I \subseteq[n-1]} A_{I}^{\triangleleft}(s)$ now follows from (2.13). This concludes the proof of Theorem 1.2 . 
2.2. Proof of Corollary 1.12, The proof of Theorem 1.2 expresses the relevant local submodule zeta functions in terms of $\mathfrak{p}$-adic integrals which are known to be representable by formulae of Denef-type. In other words, there exist algebraic varieties $V_{i}$, defined over $\mathcal{O}$, and rational functions $W_{i}(X, Y) \in \mathbb{Q}(X, Y)$ for $i=1, \ldots, M$, such that the following holds. For almost all $\mathfrak{p}$ and all $\mathfrak{O}$ we have

$$
\zeta_{\mathcal{E}(\mathfrak{O}) \curvearrowright \mathcal{L}(\mathfrak{O})}(s)=\sum_{i=1}^{M}\left|\overline{V_{i}}(\mathfrak{O} / \mathfrak{P})\right| W_{i}\left(q^{f}, q^{-f s}\right)=\frac{P_{\mathfrak{O}}\left(q^{-f s}\right)}{Q_{\mathfrak{O}}\left(q^{-f s}\right)}
$$

for coprime polynomials $P_{\mathfrak{O}}(Z), Q_{\mathfrak{O}}(Z) \in \mathbb{Z}[Z]$, say

$$
P_{\mathfrak{O}}(Z)=\sum_{i=0}^{\operatorname{deg}_{Z} P_{\mathfrak{O}}} \alpha_{i} Z^{i}, \quad Q_{\mathfrak{O}}(Z)=\prod_{j=1}^{r}\left(1-q^{f a_{j}} Z^{b_{j}}\right)=\sum_{i=0}^{\operatorname{deg}_{Z} Q_{\mathfrak{O}}} \beta_{i} Z^{i}
$$

for integers $a_{j} \in \mathbb{N}_{0}, b_{j} \in \mathbb{N}, j \in[r]$. Note that the coefficients $\beta_{i}$ of $Q_{\mathfrak{O}}$ are uniformly given by polynomials in $q^{f}$ with integral coefficients which are independent of $\mathfrak{O}$. (The pair $(\mathcal{L}, \mathcal{E})$ is almost uniform if and only the same holds for the coefficients $\alpha_{i}$ of $P_{\mathfrak{D}}$.) Moreover, the degree $\delta_{2}:=\operatorname{deg}_{Z} Q_{\mathfrak{O}}$ is independent of $\mathfrak{O}$. Note that $\alpha_{0}=\beta_{0}=1$, as the generating series $\zeta_{\mathcal{E}(\mathfrak{O}) \curvearrowright \mathcal{L}(\mathfrak{O})}(s)$ has evidently constant term 1 . The functional equation (1.3) implies that $\delta_{1}:=\operatorname{deg}_{Z} P_{\mathfrak{O}}$, too, is independent of $\mathfrak{O}$ and there exist $c_{1}, c_{2} \in \mathbb{N}_{0}$, $\sigma_{1}, \sigma_{2} \in\{0,1\}$ satisfying $c_{2}-c_{1}=\left(\begin{array}{l}n \\ 2\end{array}\right)$ and $\sigma_{1}+\sigma_{2} \equiv n \bmod (2)$ such that

$$
\begin{array}{ll}
\left.\alpha_{i}\right|_{q \rightarrow q^{-1}}=(-1)^{\sigma_{1}} q^{-f c_{1}} \alpha_{\delta_{1}-i}, & \text { for } i \in\left[\left\lfloor\delta_{1} / 2\right\rfloor\right], \\
\left.\beta_{i}\right|_{q \rightarrow q^{-1}}=(-1)^{\sigma_{2}} q^{-f c_{2}} \beta_{\delta_{2}-i}, & \text { for } i \in\left[\left\lfloor\delta_{2} / 2\right\rfloor\right],
\end{array}
$$

and $\delta_{1}-\delta_{2}=\operatorname{deg}_{q^{-f s}}\left(\zeta_{\mathcal{E}(\mathfrak{O}) \curvearrowright \mathcal{L}(\mathfrak{O})}(s)\right)=-\sum_{i=0}^{c-1} N_{i}$. Corollary 1.12 follows.

\section{NECESSARY VS. SUFFICIENT CONDITIONS FOR LOCAL FUNCTIONAL EQUATIONS}

Theorem 1.2 gives sufficient criteria for generic local functional equations for submodule zeta functions associated to nilpotent algebras of endomorphisms. Moreover, all examples of such submodule zeta functions for which we know explicit formulae for the generic Euler factors are consistent with the speculation that the hypotheses of Theorem 1.2 are also necessary for local functional equations of the form (1.3) to hold. An analogy with Igusa's local zeta function, however, sketched in Section 3.1, suggests caution. An insufficient but potentially necessary criterion for local functional equations for zeta functions in terms of so-called reduced zeta functions is discussed in Section 3.2 .

3.1. Igusa's local zeta function. The proof of Theorem 1.2 is ultimately inspired by Denef's and Meuser's proof of a functional equation for Igusa's local zeta functions associated to a homogeneous polynomial. Let $F \in \mathcal{O}\left[X_{1}, \ldots, X_{n}\right]$, let $\mathfrak{p}$ be a nonzero prime ideal of $\mathcal{O}$ of index $q$ in $\mathcal{O}$ and $\mathfrak{O}$ be a finite extension of $\mathcal{O}_{\mathfrak{p}}$, with maximal ideal $\mathfrak{P}$ of index $q^{f}$ in $\mathfrak{O}$. Igusa's local zeta function associated to $F$ at $\mathfrak{P}$ is

$$
Z_{F, \mathfrak{P}}(s)=\int_{\mathfrak{O}^{n}}|F(\boldsymbol{x})|_{\mathfrak{P}}^{s}|\mathrm{~d} \boldsymbol{x}|
$$

where ||$_{\mathfrak{P}}$ is the $\mathfrak{P}$-adic norm and $|\mathrm{d} \boldsymbol{x}|$ denotes the additive Haar measure on $\mathfrak{O}^{n}$, normalized such that $\mathfrak{O}^{n}$ has measure 1 . It is a rational function in $q^{-f s}$. If $F$ is homogeneous of degree $d$, then, for almost all $\mathfrak{p}$ and all $\mathfrak{O}$, the functional equation

$$
\left.Z_{F, \mathfrak{P}}(s)\right|_{q \rightarrow q^{-1}}=q^{-f d s} Z_{F, \mathfrak{P}}(s)
$$


holds; cf. [9, Theorem 4]. (Note that in [9] the inertia degree $f=f\left(\mathfrak{O}, \mathcal{O}_{\mathfrak{p}}\right)$ is denoted by e.) Here, as in Theorem [1.2, equation (3.1) is explained in terms of a suitable formula of the form (1.6) and the Weil conjectures for smooth projective algebraic varieties over finite fields; cf. Remark 1.7. Projectivity of the relevant varieties is a consequence of the homogeneity of $F$. This line of argument breaks down if $F$ is not homogeneous (and does not become homogeneous after an affine transformation of polynomial variables), and simple examples illustrate that functional equations such as (3.1) will not hold in general. We do not know of general necessary conditions.

Note that the functional equation (3.1) implies an analogue of (1.9), viz. that

$$
\operatorname{deg}_{q^{-f s}}\left(Z_{F, \mathfrak{P}}(s)\right)=-d,
$$

a fact first proven in [7]. We are not aware of general results on the degree of Igusa's local zeta function for nonhomogeneous polynomials, but again simple examples show that (3.2) is not universal. This contrasts with Conjecture 1.11, which predicts the degree of generic local submodule zeta functions associated with nilpotent endomorphism algebras, regardless of whether or not they satisfy functional equations.

3.2. Reduced zeta functions. In [14], Evseev introduced "reduced zeta functions" associated to various enumeration problems pertaining to finite-dimensional Lie algebras. His constructions apply quite generally to zeta functions $Z(s)=\prod_{\mathfrak{p}} Z_{\mathfrak{p}}(s)$ which satisfy Euler products, indexed by the nonzero prime ideals $\mathfrak{p}$ of a number ring, with Euler factors which are rational generating functions in $q^{-s}$ whose coefficients can be interpreted in terms of suitably "geometric functions" in the respective residue fields, such as formulae of the form (1.6). Informally speaking, the reduced zeta function $Z_{\mathrm{red}}(T)$ is a rational function in $T$ obtained by "setting $q=1$ " in such formulae, whilst treating the parameter $q^{-s}$ as an independent formal variable $T$. If $Z(s)$ is "almost uniform" (cf. Definition 1.10), i.e. if there exists $W \in \mathbb{Q}(X, Y)$ such that $Z_{\mathfrak{p}}(s)=W\left(q, q^{-s}\right)$ for almost all $\mathfrak{p}$, then $Z_{\text {red }}(T)=W(1, T)$. The formal definition given in [14] uses Euler-Poincaré characteristics in a motivic setting. We remark that the concept of reduced zeta function seems subtly related to the concept of topological zeta function; cf. [23].

Under certain conditions, reduced zeta functions satisfy functional equations upon inversion of $T$ which reflect geometric properties of polyhedral cones. Indeed, in [14], Evseev describes sufficient conditions for a reduced zeta function to be the Hilbert series $H(T)$ of a graded ring $R_{\mathcal{C}}$ associated to a rational polyhedral cone $\mathcal{C} \subseteq \mathbb{R}_{\geq 0}^{n}$; cf. [14, Proposition 4.1]. Up to a sign, the rational function resulting from the inversion of $T$ is the Hilbert series of the graded ring $R_{\mathcal{C}}$ associated to the interior of $\mathcal{C}$. A functional equation of the form

$$
H(1 / T)= \pm T^{\beta} H(T)
$$

occurs if and only if $\mathcal{C}$ has a unique minimal integral interior vector. For further details and an interpretation of these facts in terms of commutative algebra, viz. the language of Cohen-Macaulay and Gorenstein rings and modules, see [30, Chapter 1]. For an application in the context of ideal zeta functions of nilpotent Lie lattices, see Section 4.3.

A functional equation for the reduced zeta function is, in general, not sufficient for generic p-adic functional equations; cf. Example 4.15. If, however, $Z(s)$ is almost uniform as defined above, then a functional equation for the reduced zeta function $Z_{\text {red }}(T)$ is a necessary condition for functional equations for the p-adic zeta functions $Z_{\mathfrak{p}}(s)=W\left(q, q^{-s}\right)$. We speculate that this also holds without the hypothesis of almost uniformity.

In the light of the analogy with the theory of Hilbert series associated to rational polyhedral cones sketched above, it is tempting to interpret the result of the $\mathfrak{p}$-adic zeta function obtained by "inverting $q$ " in terms of a generating function enumerating "interior points", 
too. In this spirit, in [13, Definition 4.26] du Sautoy and Woodward introduced the notion of $p$-ideal of a $\mathbb{Z}_{p}$-Lie lattice, in analogy to the notion of interior point of a polyhedral cone. Their hope clearly was to interpret local functional equations in terms of natural correspondences between the ideal- and $p$-ideal-lattices. Our proof of Theorem 1.2, however, relies in an essential manner on geometric properties of smooth projective algebraic varieties, as established by the Weil conjectures (see Remark 1.7). It seems therefore that any such interpretation of the local functional equations established in Theorem 1.2 would have to interpolate between these deep algebro-geometric symmetries on the one hand and the symmetries satisfied by Hilbert series associated to cones on the other.

\section{IDEAL ZETA FUnCTIONS OF NILPOTENT LiE LATTICES}

In this section we discuss applications of Theorem 1.2 to ideal zeta functions of nilpotent Lie lattices. Ideal zeta functions of nilpotent Lie rings have been introduced in [16] as tools in the study of normal subgroup growth of finitely generated nilpotent groups. The technical tool facilitating this linearization is the Mal'cev correspondence mentioned in Section 1.2. Hence, all results in this section on ideal zeta functions of nilpotent Lie lattices have immediate consequences on normal subgroup zeta functions of finitely generated nilpotent groups. Only in Corollary 1.9) did we choose to spell out such a consequence (of Theorem 4.4, in this instance).

Assume that $\mathcal{L}$ is a nilpotent $\mathcal{O}$-Lie lattice satisfying Assumption 1.1-which, as we saw in Remark 1.1, is vacuous for Lie rings (i.e. if $\mathcal{O}=\mathbb{Z}$ ) -, with Lie bracket [,]. Recall that $\left(Z_{i}\right)_{i=0}^{c}$ is the upper central series of $\mathcal{L}$. In particular, $c$ is the nilpotency class of $\mathcal{L}$ and $N_{i}=\operatorname{rk}_{\mathcal{O}}\left(\mathcal{L} / Z_{i}\right)$ for all $i \in[c]_{0}$. As noted in Remark 1.8, Condition 1.1 is trivially satisfied if $c \leq 2$. In these cases, Theorem 1.2 confirms known results. For $c=1$, see Example 1.4. For $c=2$, Theorem 1.2 in this setting is (a mild generalization of) [35, Theorem C].

4.1. Examples without functional equations. That local functional equations akin to (1.3) may fail in nilpotency class greater than 2 was first discovered by Woodward.

Example 4.1. Consider the class-4-nilpotent Lie ring

$$
\mathcal{L}=\mathrm{Fil}_{4}=\left\langle z, x_{1}, x_{2}, x_{3}, x_{4} \mid\left[z, x_{1}\right]=x_{2},\left[z, x_{2}\right]=x_{3},\left[z, x_{3}\right]=x_{4},\left[x_{1}, x_{2}\right]=x_{4}\right\rangle_{\mathbb{Z}} .
$$

Here, as well as in comparable Lie lattice presentations throughout the paper, products among generators other than those following - by antisymmetry or the Jacobi identity from the given ones are assumed to be trivial. In the given example this means that $x_{4}$ is central and $x_{3}$ commutes with both $x_{1}$ and $x_{2}$. [13, Theorem 2.39] gives explicit formulae for the local ideal zeta functions $\zeta_{\mathrm{Fil}_{4}\left(\mathbb{Z}_{p}\right)}(s)$, valid for all primes $p$. They are all given by a single rational function $W(X, Y) \in \mathbb{Q}(X, Y)$ upon the substitution $X=p, Y=p^{-s}$ and do not satisfy a functional equation of the form (1.3). The associative algebra $\mathcal{E}$ generated by $\operatorname{ad}\left(\mathrm{Fil}_{4}\right)$ does not satisfy the conditions of Theorem 1.2. Indeed, it is generated by $\operatorname{ad}(z)$ and $\operatorname{ad}\left(x_{1}\right)$ which, with respect to the chosen cocentral $\mathbb{Z}$-basis $\left(z, x_{1}, x_{2}, x_{3}, x_{4}\right)$ of $\mathrm{Fil}_{4}$ are represented by the integral $5 \times 5$-matrices

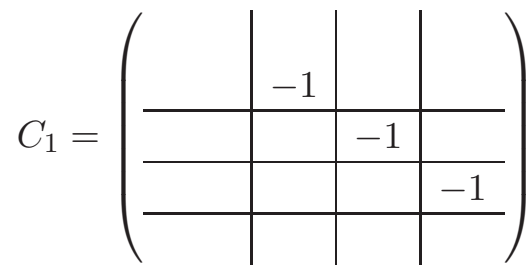

and

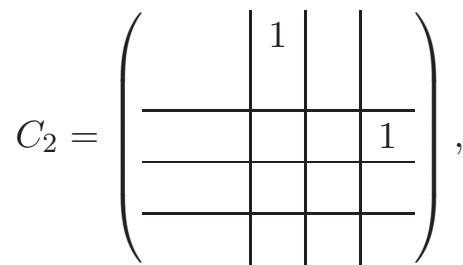

respectively. Here, the block structure reflects the decomposition $\mathcal{L}=\bigoplus_{i=1}^{4} \mathcal{L}_{i}$ with $\mathcal{L}_{1}=\left\langle z, x_{1}\right\rangle_{\mathbb{Z}}$ and $\mathcal{L}_{i}=\left\langle x_{i}\right\rangle_{\mathbb{Z}}$ for $i \in\{2,3,4\}$. The matrix $C_{2}$ is not as prescribed in 
Condition 2.1, as it is not supported solely on the first block-off diagonal: $\left(C_{2}\right)_{34} \neq 0$. The failure of the functional equation reflects the (easily verifiable) fact that no other decomposition or choice of generators for $\mathcal{E}$ mitigates this failure.

Example 4.2. Consider the class-3-nilpotent Lie ring

$$
\mathcal{L}=\mathfrak{g}_{6,6}=\left\langle x_{1}, \ldots, x_{6} \mid\left[x_{1}, x_{2}\right]=x_{4},\left[x_{1}, x_{3}\right]=x_{5},\left[x_{1}, x_{4}\right]=x_{6},\left[x_{2}, x_{3}\right]=x_{6}\right\rangle_{\mathbb{Z}} .
$$

As observed in [13, Example 4.58], $Z_{1}=\left\langle x_{5}, x_{6}\right\rangle_{\mathbb{Z}}, Z_{2}=\left\langle x_{3}, x_{4}, x_{5}, x_{6}\right\rangle_{\mathbb{Z}}, Z_{3}=\mathcal{L}$. Set $\mathcal{L}_{1}=\left\langle x_{1}, x_{2}\right\rangle_{\mathbb{Z}}, \mathcal{L}_{2}=\left\langle x_{3}, x_{4}\right\rangle_{\mathbb{Z}}, \mathcal{L}_{3}=\left\langle x_{5}, x_{6}\right\rangle_{\mathbb{Z}}$. Whilst $c_{1}=\operatorname{ad}\left(x_{1}\right)$ and $c_{2}=\operatorname{ad}\left(x_{2}\right)$ satisfy condition (1.2), $c_{3}=\operatorname{ad}\left(x_{3}\right)$ does not. Moreover, this failure is independent of the specific choice of complements $\mathcal{L}_{i}$. Crucially, $c_{3}$ is not contained in the associative algebra generated by $c_{1}$ and $c_{2}$. This is consistent with the fact that the ideal zeta function of $\mathfrak{g}_{6,6}$ does not satisfy the conclusions of Theorem [1.2, see [13, Theorem 2.44].

Remark 4.3. Like many others of their kind, the computations in 13 are only carried out for local rings $\mathfrak{o}=\mathbb{Z}_{p}$. The resulting formulae, however, also cover the case of general finite extensions $\mathfrak{o}$ of $\mathbb{Z}_{p}$; one replaces $p$ by the residue field cardinality $q$. See [21, Section 2.3] for a formal justification of this stability under local base extension.

Numerous further formulae for local ideal zeta functions of nilpotent Lie rings lacking a generic local functional equation can be found in [13, Section 2]. Like most formulae recorded in [13, they are obtained by computations with $p$-adic cone integrals; cf. [11]. In [13. Conjecture 4.5] du Sautoy and Woodward formulate a sort of conjecture on such cone integrals that would imply functional equations akin to (1.3). The conjecture's hypotheses, however, suffer from a degree of illdefinedness ("Suppose that [two specified conditions] and some as-yet-undetermined conditions hold."). It takes indeed a "cavalier attitude to the incompleteness of Conjecture 4.5" ([13, p. 98]) to use it to speculate about the occurrence of local functional equations of ideal zeta functions of Lie rings.

The shortcomings of [13, Conjecture 4.5] notwithstanding, the present paper owes a great deal of inspiration to [13, Chapter 4]. Our very Condition 1.1 is modelled on the conjunction of the properties $(\dagger)$ and $(*)$ in [13, Definition 4.56]; our matrix $\delta$ (see (2.6) ) represents - up to a scalar factor - the map $\pi_{\mathcal{B}}$ in [13, Definition 4.40] with respect to a cocentral basis, a concept generalizing [13, Definition 4.37]. There are, nonetheless, two fundamental differences in approach. Firstly, we do not analyze cone integrals but develop the $\mathfrak{p}$-adic machinery introduced in 35. Secondly, we realize that the ideal zeta function of a nilpotent Lie lattice $\mathcal{L}$ is determined by the associative algebra generated by $\operatorname{ad}(\mathcal{L})$; rather than hypothesizing about linear bases for the former, we formulate necessary conditions on suitable generators of the latter.

In the remainder of the section we develop a number of (unconditional) applications of Theorem 1.2 establishing generic local functional equations for ideal zeta functions of nilpotent Lie lattices, confirming some of the more specific conjectures in [13]. We record these results in the characteristic-independent fashion made possible by Corollary [1.3. Recall that, given a Lie ring (viz. $\mathbb{Z}$-Lie lattice) $\mathcal{L}$ and a compact discrete valuation ring $\mathfrak{o}$ (of arbitrary characteristic), the ideal zeta function $\zeta_{\mathcal{L}(\mathfrak{o})}^{\triangleleft}(s)$ of $\mathcal{L}(\mathfrak{o})=\mathcal{L} \otimes_{\mathbb{Z}} \mathfrak{o}$ is the rational ordinary generating function enumerating the o-ideals of $\mathcal{L}(\mathfrak{o})$ of finite additive index in $\mathcal{L}(\mathfrak{o})$.

4.2. Free nilpotent Lie rings. Given $c, d \in \mathbb{N}$, consider the free class-c-nilpotent Lie ring $\mathfrak{f}_{c, d}$ on free Lie generators $x_{1}, \ldots, x_{d}$. The well-known formula for the $\mathbb{Z}$-ranks of the sections of the terms $\gamma_{j}\left(\mathfrak{f}_{c, d}\right)$ of the lower central series of $\mathfrak{f}_{c, d}$ (which coincides with the 
upper central series of $\mathfrak{f}_{c, d}$ ), due to Witt ([36, Satz 3]), implies that, for $i \in[c]_{0}$,

$$
\operatorname{rk}_{\mathbb{Z}}\left(\mathfrak{f}_{c, d} / \gamma_{c-i+1}\left(\mathfrak{f}_{c, d}\right)\right)=\sum_{1 \leq j \leq c-i} \frac{1}{j} \sum_{k \mid j} \mu(k) d^{j / k}=N_{i},
$$

the numbers defined in (1.7). The following consequence of Theorem 1.2 and its Corollary 1.3 implies the conclusion of [13, Theorem 1.3] without relying on the incomplete [13, Conjecture 4.5].

Theorem 4.4. For almost all primes $p$ and all compact discrete valuation rings of residue characteristic $p$,

$$
\left.\zeta_{\mathfrak{f}_{c, d}(\mathfrak{o})}^{\triangleleft}(s)\right|_{p \rightarrow p^{-1}}=(-1)^{N_{0}} q^{\left(\begin{array}{c}
N_{0} \\
2
\end{array}\right)-s\left(\sum_{i=0}^{c-1} N_{i}\right)} \zeta_{\mathfrak{f}_{c, d}(\mathfrak{o})}(s) .
$$

Proof. We choose a Hall $(\mathbb{Z}-)$ basis $\mathcal{H}$ on $\left\{x_{1}, \ldots, x_{d}\right\}$ for $\mathfrak{f}_{c, d}$; cf. [19. By construction, elements of $\mathcal{H}$ are Lie monomials in $x_{1}, \ldots, x_{d}$ with a well-defined degree, or weight $i \in$ $[c]$. For $i \in[c]$, write $\mathcal{H}^{i}$ for the set of elements of $\mathcal{H}$ of weight $i$. For instance, $\mathcal{H}^{1}=$ $\left\{x_{1}, \ldots, x_{d}\right\}$. Note that $\bigcup_{\iota<i} \mathcal{H}^{\iota}$ has cardinality $N_{i}$ for each $i \in[c]_{0}$. Setting $\mathcal{L}_{i}:=\left\langle\mathcal{H}^{i}\right\rangle_{\mathbb{Z}}$ for $i \in[c]$ and $\left\{c_{1}, \ldots, c_{d}\right\}=\left\{\operatorname{ad}\left(x_{1}\right), \ldots, \operatorname{ad}\left(x_{d}\right)\right\}$, Condition 1.1 is clearly satisfied. The result thus follows from Theorem 1.2 and Corollary 1.3 .

Explicit formulae for $\zeta_{\mathfrak{f}_{c, d}(\mathfrak{o})}(s)$ are known for $c=1$ (see (1.5)), $c=2$ (see [34]), and $(c, d)=(3,2)($ see [13, Theorem 2.35]).

Remark 4.5. Theorem 4.4 also implies a corrected version of the conclusion of [13, Theorem 4.73]. Note that the meaning of the numbers $N_{1}$ and $N_{2}$ there is different from the one in the present paper. In any case, the power of $p^{-s}$ on the right-hand side of 13 , (4.45)] is not equal to the sum of the coranks of the upper central series of $\mathfrak{f}_{c, d}$.

4.3. Some Lie rings of maximal class and their amalgams. Given an (integer) partition $\lambda=\left(\lambda_{1}, \ldots, \lambda_{r}\right) \in \mathbb{N}^{r}$, with $\lambda_{1} \geq \lambda_{2} \geq \cdots \geq \lambda_{r}$, define the class- $\lambda_{1}$-nilpotent Lie ring

$$
\mathcal{L}_{\lambda}=\left\langle x_{0},\left\{x_{i, j}\right\}_{i \in[r], j \in\left[\lambda_{i}\right]} \mid \forall i \in[r], j \in\left[\lambda_{j}-1\right]:\left[x_{0}, x_{i, j}\right]=x_{i, j+1}\right\rangle_{\mathbb{Z}} .
$$

For $r=1$ and $\lambda_{1} \geq 2$ this yields the Lie ring $M_{\lambda_{1}}$ of maximal class $\lambda_{1}$ described in [13, p. 99]. If $\lambda_{r} \geq 2$, then $\mathcal{L}_{\lambda}$ is obtained from amalgamating $M_{\lambda_{i}}, i \in[r]$, along $x_{0}$. If $\lambda=1^{(r)}=(1, \ldots, 1)$, then we obtain the abelian Lie ring $\mathbb{Z}^{1+r} ;$ cf. Example 1.4. The general case is evidently just an amalgamation of these special cases, again along $x_{0}$. For $\lambda=2^{(r)}$ we get "Grenham's Lie rings" $\mathcal{G}_{1+r}$ on $1+r$ Lie generators; cf. [13, Section 2.6]. Explicit formulae for the local ideal zeta functions $\zeta_{\mathcal{L}_{2(r)}\left(\mathbb{Z}_{p}\right)}(s)$, valid for all $p$, are given in [33, Theorem 5]. (On the face of it, the formulae there are for the local normal zeta functions of the torsion-free finitely generated nilpotent groups associated to the Lie rings $\mathcal{L}_{2(r)}$ by the Mal'cev correspondence. As $c=2$, however, the formulae coincide with those of the ideal zeta functions of $\mathcal{L}_{2^{(r)}}\left(\mathbb{Z}_{p}\right)$ for all $p$; cf. [16, Remark on p. 206].)

Definition 4.6. We say that $\lambda$ is a near rectangle if it is of the form $\lambda=\left(c^{\left(r_{1}\right)}, 1^{\left(r_{2}\right)}\right)$ for some $c \in \mathbb{N}$ and $r_{1}, r_{2} \in \mathbb{N}_{0}$.

A geometric interpretation of this property of $\lambda$ is given in Proposition 4.12

Lemma 4.7. Condition 1.1 is satisfiable for $\mathcal{L}_{\lambda}$ if and only if $\lambda$ is a near rectangle. 
Proof. We may assume that $c>1$. If $\lambda=\left(c^{\left(r_{1}\right)}, 1^{\left(r_{2}\right)}\right)$ is a near rectangle, then, setting

$$
\mathcal{L}_{i}= \begin{cases}\left\langle x_{0}, x_{1,1}, \ldots, x_{r_{1}, 1}\right\rangle_{\mathbb{Z}} & \text { for } i=1, \\ \left\langle x_{1, i}, \ldots, x_{r_{1}, i}\right\rangle_{\mathbb{Z}} & \text { for } i \in] 1, c-1], \\ \left\langle x_{1, c}, \ldots, x_{r_{1}, c}, x_{r_{1}+1,1}, \ldots x_{r_{1}+r_{2}, 1}\right\rangle_{\mathbb{Z}} & \text { for } i=c,\end{cases}
$$

and $\left\{c_{1}, \ldots, c_{d}\right\}=\left\{\operatorname{ad}\left(x_{0}\right), \operatorname{ad}\left(x_{i, 1}\right) \mid i \in\left[r_{1}\right]\right\}$, Condition 1.1 is satisfied.

Assume now that $\lambda$ is not a near rectangle. This implies that $c>2$ and there exists $k \in[r-1]$ which is minimal with respect to the property that $c=\lambda_{k}>\lambda_{k+1}>1$. Write $\mathcal{L}=\mathcal{L}_{\lambda}$, with upper central series $\left(Z_{i}\right)_{i=0}^{c}$. Following [13, Definition 4.49], we define the depth of an element $x \in \mathcal{L}$ as

$$
\operatorname{dep}(x)=c+1-\min \left\{i \in[c]_{0} \mid x \in Z_{i}\right\} .
$$

The depth-1-elements of the $\mathbb{Z}$-basis $\mathcal{B}=\left\{x_{0}, x_{i, j} \mid i \in[r], j \in\left[\lambda_{i}\right]\right\}$ of $\mathcal{L}$ are exactly $x_{0}$ and $x_{\kappa, 1}$, for $\kappa \in[k]$. To see that Condition 1.1 is not satisfiable, note that the subalgebra $\mathcal{E}_{1}=\langle\operatorname{ad}(z) \mid z \in \mathcal{B}, \operatorname{dep}(z)=1\rangle$ of the associative algebra $\mathcal{E}$ generated by $\operatorname{ad}(\mathcal{L})$ is a proper subalgebra of $\mathcal{E}$; indeed, the element $x_{k+1,1}$ has depth $c-\lambda_{k+1}+1>1$ and $\operatorname{ad}\left(x_{k+1,1}\right) \in \mathcal{E} \backslash \mathcal{E}_{1}$. This follows, for instance, from the fact that $\mathcal{B}$ clearly has the property that if $z, z^{\prime} \in \mathcal{B}$, then either $\left[z, z^{\prime}\right]=0$ or $\operatorname{dep}\left[z, z^{\prime}\right]=\operatorname{dep}(z)+\operatorname{dep}\left(z^{\prime}\right)$.

4.3.1. Local ideal zeta functions. The following consequence of Theorem 1.2 implies, in particular, the first of the two conclusions of [13, Proposition 4.75]. Note that the latter only considers the case that $\lambda_{r} \geq 2$ and is conditional on the incomplete [13, Conjecture 4.5].

Theorem 4.8. Let $c \in \mathbb{N}$ and $r_{1}, r_{2} \in \mathbb{N}_{0}$. For almost all primes $p$ and all compact discrete valuation rings of residue characteristic $p$,

$$
\left.\left.\zeta_{\mathcal{L}_{\left(c\left(r_{1}\right), 1^{(}\left(r_{2}\right)\right)}^{\triangleleft}(\mathfrak{o})}(s)\right|_{p \rightarrow p^{-1}}=(-1)^{1+c r_{1}+r_{2}} q^{\left(\begin{array}{c}
1+c r_{1}+r_{2} \\
2
\end{array}\right)-s\left(c+\left(\begin{array}{c}
c+1 \\
2
\end{array}\right) r_{1}+r_{2}\right)} \zeta_{\left.\mathcal{L}_{(c}\left(r_{1}\right), 1\left(r_{2}\right)\right)}(\mathfrak{o})\right) .
$$

In particular, 13, Conjecture 4.24] about the Lie rings $M_{c}$ of maximal class $c$ holds.

Proof. By Lemma 4.7, Condition 1.1 is satisfied. We find $\operatorname{rk}_{\mathbb{Z}}\left(\mathcal{L}_{\left(c^{\left(r_{1}\right)}, 1^{\left(r_{2}\right)}\right)}\right)=N_{0}=1+$ $c r_{1}+r_{2}$ and, more generally, $N_{i}=1+(c-i) r_{1}+r_{2} \delta_{i=0}$ for $i \in[c-1]_{0}$, whence $\sum_{i=0}^{c-1} N_{i}=$ $c+\left(\begin{array}{c}c+1 \\ 2\end{array}\right) r_{1}+r_{2}$. The result thus follows from Theorem 1.2 and Corollary 1.3 .

The second, conjectural conclusion of [13, Proposition 4.75] suggests a positive answer to the following question.

Question 4.9. Is the "near rectangle" condition on $\lambda$ necessary for local functional equations for the ideal zeta functions of the Lie rings $\mathcal{L}_{\lambda}$ ?

A positive answer might also be hinted at by the explicit formulae for $\lambda=(3,2)$ - the smallest partition which is not a near rectangle - given in [13, Theorem 2.32] as well as the following results on the reduced zeta functions $\zeta_{\mathcal{L}_{\lambda} \text {,red }}^{\triangleleft}(T)$; cf. Section 3.2 .

4.3.2. Reduced ideal zeta functions. Let $\mathcal{L}$ be a $\mathbb{C} \llbracket T \rrbracket$-Lie algebra with $\mathbb{C} \llbracket T \rrbracket$-basis $\mathcal{B}=$ $\left\{b_{1}, \ldots, b_{d}\right\}$. In [14, the basis $\mathcal{B}$ is called simple if, for all $1 \leq i, j \leq d$, there exist $\ell \in[d]$ and $a \in \mathbb{C} \llbracket T \rrbracket$ such that $\left[b_{i}, b_{j}\right]=a b_{\ell}$. A pair $\left(b_{i}, b_{j}\right)$ of basis vectors is called removable if there exist integers $\ell_{1}, \ldots, \ell_{d}$ with $\ell_{i} \neq \ell_{j}$ such that, for all $z \in \mathbb{C} \backslash\{0\}$ and all $r \in[d]$, the maps $x_{r} \mapsto z^{\ell_{r}} x_{r}$ are automorphisms of $\mathcal{L}$. (Strictly speaking, 14] defines removability only for pairs of indices of basis elements.) Finally, the basis $\mathcal{B}$ is called nice if all pairs $\left(b_{i}, b_{j}\right)$ are removable; cf. [14, Theorem 3.2] which establishes that this characterization is equivalent to the definition of niceness in [14, Section 4]. 
Proposition 4.10. The $\mathbb{C} \llbracket T \rrbracket$-Basis $\mathcal{B}_{\lambda}=\left\{x_{0}, x_{i, j} \mid i \in[r], j \in\left[\lambda_{i}\right]\right\}$ of the $\mathbb{C} \llbracket T \rrbracket$-Lie algebra $\mathcal{L}_{\lambda}(\mathbb{C} \llbracket T \rrbracket)$ is nice and simple in the sense of 14$]$.

Proof. $\mathcal{B}_{\lambda}$ is simple as, for all $x, y \in \mathcal{B}_{\lambda}$, there exist $z \in \mathcal{B}_{\lambda}$ and $\varepsilon \in\{0,1,-1\}$ such that $[x, y]=\varepsilon z$. To see that $\mathcal{B}_{\lambda}$ is nice we show that all pairs $(x, y) \in \mathcal{B}_{\lambda}^{2}$ with $x \neq y$ are removable. Concretely, we need to find pairwise distinct integers $l_{0}, l_{i, j}, i \in[r], j \in\left[\lambda_{i}\right]$, such that, for all $z \in \mathbb{C} \backslash\{0\}$, the maps

$$
x_{0} \mapsto z^{l_{0}} x_{0}, \quad x_{i, j} \mapsto z^{l_{i, j}} x_{i, j}, \quad i \in[r], j \in\left[\lambda_{i}\right],
$$

are automorphisms of $\mathcal{L}_{\lambda}(\mathbb{C} \llbracket T \rrbracket)$. This is the case whenever $l_{0}+l_{i, j}=l_{i, j+1}$ for all $i \in[r]$, $j \in\left[\lambda_{i}-1\right]$. Setting $l_{0}=1$ and $l_{i, j}=1+\left(\sum_{\iota<i} \lambda_{\iota}\right)+j$ is one way to achieve this.

Define the rational polyhedral cone

$$
\mathcal{C}_{\lambda}=\left\{\mathbf{n} \in \mathbb{R}_{\geq 0}^{1+\sum_{i=1}^{r} \lambda_{i}} \mid \forall i \in[r]: n_{0}, n_{i, 1} \geq n_{i, 2} \geq \cdots \geq n_{i, \lambda_{i}}\right\} .
$$

Given $\mathbf{n}=\left(n_{1}, \ldots, n_{h}\right) \in \mathbb{R}^{h}$ for some $h \in \mathbb{N}$, we set $\sum \mathbf{n}=\sum_{j=1}^{h} n_{j}$.

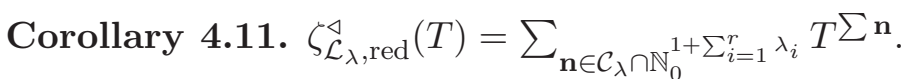

Proof. As $\mathcal{B}_{\lambda}$ is nice and simple, this follows from [14, Proposition 4.1], where $\mathcal{C}_{\lambda}$ is called $\mathcal{C}_{\mathcal{B}_{\lambda}}^{\triangleleft}$

The reduced zeta function $\zeta_{\mathcal{L}_{\lambda} \text {,red }}(T)$ is thus the Hilbert series associated to the graded monoid algebra $R_{\mathcal{C}_{\lambda}}$ spanned by the integral points in $\mathcal{C}_{\lambda}$. Such Hilbert series satisfy functional equations upon inversion of $T$ if and only if (!) $R_{\mathcal{C}_{\lambda}}$ is Gorenstein (cf. [30, Theorem 12.7]). The latter condition is satisfied if and only if there exists a unique minimal integral vector $\boldsymbol{\beta}$ in the interior $\mathcal{C}_{\lambda}^{\circ}$ of $\mathcal{C}_{\lambda}$, i.e.

$$
\text { if } \gamma \in \mathcal{C}_{\lambda}^{\circ} \cap \mathbb{N}^{1+\sum_{i=1}^{r} \lambda_{i}} \text {, then } \boldsymbol{\gamma}-\boldsymbol{\beta} \in \mathcal{C}_{\lambda} \text {. }
$$

Note that $\mathcal{C}_{\lambda}^{\circ}$ is defined by replacing the inequalities in (4.1) by strict inequalities.

Proposition 4.12. A unique minimal integral interior vector $\boldsymbol{\beta} \in \mathcal{C}_{\lambda}^{\circ}$ exists if and only if $\lambda$ is a near rectangle.

Proof. Assume that

$$
\boldsymbol{\beta}=\left(\beta_{0}, \beta_{1,1}, \beta_{1,2}, \ldots, \beta_{1 \lambda_{1}}, \beta_{21}, \ldots \beta_{2 \lambda_{2}}, \ldots, \beta_{r 1}, \ldots, \beta_{r \lambda_{r}}\right) \in \mathcal{C}_{\lambda}^{\circ} \cap \mathbb{N}^{1+\sum_{i=1}^{r} \lambda_{i}}
$$

has the property (4.2). It is not hard to check that

$$
\left(\beta_{0}, \beta_{1,1}, \beta_{1,2}, \ldots, \beta_{1, \lambda_{1}}\right)=\left(\lambda_{1}, \lambda_{1}, \lambda_{1}-1, \ldots, 2,1\right) .
$$

Observe that $\lambda$ is a near rectangle if and only if this property determines $\boldsymbol{\beta}$ uniquely. Indeed, if $\lambda$ is a near rectangle, say $\lambda=\left(c^{\left(r_{1}\right)}, 1^{\left(r_{2}\right)}\right)$ for some $c \in \mathbb{N}$ and $r_{1}, r_{2} \in \mathbb{N}_{0}$, then

$$
\begin{aligned}
\boldsymbol{\beta}=(c, c, c-1, \ldots, 2,1, c, c-1, \ldots, 2,1, \ldots c, c-1, \ldots, 2,1, & \left.1^{\left(r_{2}\right)}\right) \\
& \in \mathcal{C}_{\left(c^{\left(r_{1}\right)}, 1^{\left(r_{2}\right)}\right)}^{\circ} \cap \mathbb{N}^{1+c r_{1}+r_{2}}
\end{aligned}
$$

satisfies both (4.2) and (4.3) and is clearly unique with this property. If $\lambda$ is not a near rectangle, there exist more than one ways to "complete" the vector in (4.3) to an element $\beta \in \mathcal{C}_{\lambda}^{\circ} \cap \mathbb{N}^{1+\sum_{i=1}^{r} \lambda_{i}}$ such that $\beta_{0}=\lambda_{1}$ and $\beta_{i, 1}=\lambda_{1}$ for $i \in[r]$. The difference between any two of these completions, however, is zero on these coordinates, hence is zero, contradiction. 
Corollary 4.13. $\lambda$ is a near rectangle if and only if there exist $k, l \in \mathbb{Z}$ such that

$$
\zeta_{\mathcal{L}_{\lambda}, \text { red }}^{\triangleleft}(1 / T)=(-1)^{l} T^{k} \zeta_{\mathcal{L}_{\lambda}, \text { red }}^{\triangleleft}(T) .
$$

In this case, $l \equiv 1+c r_{1}+r_{2} \bmod (2)$ and $k=c+\left(\begin{array}{c}c+1 \\ 2\end{array}\right) r_{1}+r_{2}$.

Remark 4.14. If $\zeta_{\mathcal{L}_{\lambda}}^{\triangleleft}(s)$ is almost uniform, say $\zeta_{\mathcal{L}_{\lambda}\left(\mathbb{Z}_{p}\right)}(s)=W_{\lambda}\left(p, p^{-s}\right)$ for $W_{\lambda}(X, Y) \in$ $\mathbb{Q}(X, Y)$ and almost all primes $p$, then Corollary 4.13 implies that the "near rectangle condition" on $\lambda$ is also necessary for local p-adic functional equations. Indeed, in this case, $\zeta_{\mathcal{L}_{\lambda}, \text { red }}^{\triangleleft}(T)=W_{\lambda}(1, T)$; cf. [14, Section 3].

Example 4.15. A functional equation of the form (4.4) for reduced zeta functions is not, in general, sufficient for functional equations for generic $\mathfrak{p}$-adic zeta functions. Consider, for instance, the Lie rings $M_{4}=\mathcal{L}_{(4)}$ and $\mathrm{Fil}_{4}$; cf. Example 4.1. Whereas the local ideal zeta functions $\zeta_{M_{4}\left(\mathbb{Z}_{p}\right)}(s)$ satisfy functional equations (cf. Corollary 4.8), the local ideal zeta functions $\zeta_{\mathrm{Fil}_{4}\left(\mathbb{Z}_{p}\right)}^{\triangleleft}(s)$ do not (cf. [13, Theorem 2.39]). The respective reduced ideal zeta functions, however, coincide; cf. [14, Example 4.5].

\section{Further EXAMPLES}

In this section we discuss three applications of Theorem 1.2 and Corollary 1.3 to submodule zeta functions which are not ideal zeta functions of nilpotent Lie lattices. The case of one-generator matrix algebras is discussed in Section 5.1. In Section 5.2 we consider a class of abelian matrix algebras. Section 5.3 is dedicated to full algebras of strictly upper triangular matrices. Throughout, $\mathfrak{o}$ is a compact discrete valuation ring of arbitrary characteristic and residue field cardinality $q$, a power of the residue characteristic $p$, and let $n \in \mathbb{N}$.

5.1. The case $d=1$. In [25, zeta functions enumerating submodules invariant under a single, not necessarily nilpotent endomorphism of a finitely generated $\mathcal{O}$-module are studied. [25, Theorem A] gives, in particular, an explicit formula for almost all of the Euler factors of such zeta functions in terms of translates of Dedekind zeta functions of number fields. Both these number fields and the combinatorics of the translations are determined by the rational canonical form for the given endomorphism. [25, Theorem B] establishes generic functional equations for the Euler factors. In the nilpotent case, to which the general case is reduced in [25, Section 3], they confirm Theorem [1.2.

\subsection{A class of abelian matrix algebras.}

5.2.1. Local formulae. Let $p$ be a prime and o be a compact discrete valuation ring of residue characteristic $p$. We set

$$
\mathcal{M}_{\mathbf{1}}(\mathfrak{o})=\mathcal{M}_{(\underbrace{1, \ldots, 1}_{n \text { times }})}(\mathfrak{o})=\left\{\left(\begin{array}{cc}
0 & \operatorname{diag}\left(z_{1}, \ldots, z_{n}\right) \\
0 & 0
\end{array}\right) \mid z_{1}, \ldots, z_{n} \in \mathfrak{o}\right\} \leq \operatorname{Mat}_{2 n}(\mathfrak{o})
$$

and consider $\mathfrak{o}^{2 n}$ as an $\mathcal{M}_{\mathbf{1}}(\mathfrak{o})$-module by right multiplication. We record a formula for the submodule zeta functions $\zeta_{\mathcal{M}_{1}(\mathfrak{o}) \curvearrowright \mathfrak{o}^{2 n}}(s)$. It is very similar to that for the ideal zeta functions $\zeta_{H(\mathfrak{o})^{n}}^{\triangleleft}(s)$ of the $n$-fold direct product of the Heisenberg $\mathfrak{o}$-Lie lattices $H(\mathfrak{o})$ of strictly upper triangular $3 \times 3$-matrices over o given in [26, Theorem 3.1]. We formulate this result partly in the notation of [26] and note that the relevant local results in [26] are - despite their formulation in characteristic zero only - in fact valid for compact discrete valuation rings of arbitrary characteristic. In particular, $\mathcal{D}_{2 n}$ denotes the set of Dyck words 
in letters $\mathbf{0}$ and $\mathbf{1}$ of length $2 n$, of cardinality $\frac{1}{n+1}\left(\begin{array}{c}2 n \\ n\end{array}\right)$, the $n$-th Catalan number. Recall the formula (1.5) for $\zeta_{\{0\} \curvearrowright \mathfrak{o}^{n}}(s)$.

Theorem 5.1.

$$
\zeta_{\mathcal{M}_{\mathbf{1}}(\mathfrak{o}) \curvearrowright \mathfrak{o}^{2 n}}(s)=\left(1-q^{-s}\right)^{n} \zeta_{\{0\} \curvearrowright \mathfrak{o}^{n}}(s) \sum_{w \in \mathcal{D}_{2 n}} D_{w}\left(q, q^{-s}\right),
$$

where, for $w=\prod_{i=1}^{r}\left(\mathbf{0}^{L_{i}-L_{i-1}} \mathbf{1}^{M_{i}-M_{i-1}}\right) \in \mathcal{D}_{2 n}$, the rational function $D_{w}\left(q, q^{-s}\right)$ is defined as $D_{w}^{1}\left(p, p^{-s}\right)$ in [26. Theorem 3.1] with $q$ in place of $p$ and with numerical data

$$
\begin{array}{ll}
x_{j}=q^{j\left(n+L_{i}-j\right)-s\left(L_{i}+j\right)} & \text { for } \left.j \in] M_{i-1}, M_{i}\right], \\
y_{j}=q^{\left(n-M_{i-1}+j\right) M_{i-1}-s\left(j+M_{i-1}\right)} & \text { for } \left.j \in] L_{i-1}, L_{i}\right] .
\end{array}
$$

Sketch of proof. Mutatis mutandis, the analysis of [26, Theorem 3.1] carries over. The centre $Z(H(\mathfrak{o})) \cong \mathfrak{o}^{n}$ is replaced by $Z_{1}(\mathfrak{o})=\operatorname{Cent}_{\mathcal{M}_{\mathbf{1}}(\mathfrak{o})}\left(\mathfrak{o}^{2 n}\right) \cong \mathfrak{o}^{n}$, whereas the role of the cocentre $H(\mathfrak{o}) / Z(H(\mathfrak{o})) \cong \mathfrak{o}^{2 n}$ is taken by $\mathfrak{o}^{2 n} / Z_{1}(\mathfrak{o}) \cong \mathfrak{o}^{n}$, explaining the change in numerical data.

The construction of the algebra $\mathcal{M}_{\mathbf{1}}(\mathfrak{o})$ may be generalized by replacing the diagonal matrices in (5.1) by "generic block-diagonal" matrices with block sizes $f_{1}, \ldots, f_{g}$, say. Roughly speaking, formulae for the submodule zeta functions associated to the resulting nilpotent algebras

$$
\mathcal{M}_{\mathbf{f}}(\mathfrak{o})=\mathcal{M}_{\left(f_{1}, \ldots, f_{g}\right)}(\mathfrak{o})=\left(\begin{array}{cc}
0 & \operatorname{diag}\left(\operatorname{Mat}_{f_{1}}(\mathfrak{o}), \ldots, \operatorname{Mat}_{f_{g}}(\mathfrak{o})\right) \\
0 & 0
\end{array}\right) \leq \operatorname{Mat}_{2 n}(\mathfrak{o}),
$$

acting on $\mathfrak{o}^{2 n}$ by right multiplication, are obtained by modifying the "numerical data" in the formulae given in [26, Theorem 3.6] and in the formula for the zeta function preceding it. We leave the precise details to the reader, spelling out only the result in the other "extremal" case $\mathbf{f}=(n)$, yielding

$$
\mathcal{M}_{(n)}(\mathfrak{o})=\left(\begin{array}{cc}
0 & \operatorname{Mat}_{n}(\mathfrak{o}) \\
0 & 0
\end{array}\right) \leq \operatorname{Mat}_{2 n}(\mathfrak{o})
$$

The following is analogous to [26, Corollary 3.7].

Theorem 5.2.

$$
\zeta_{\mathcal{M}_{(n)}(\mathfrak{o}) \curvearrowright \mathfrak{o}^{2 n}}(s)=\zeta_{\{0\} \curvearrowright \mathfrak{o}^{n}}(s) \frac{1}{1-x_{n}} \sum_{I \subseteq[n-1]}\left(\begin{array}{c}
n \\
I
\end{array}\right)_{q^{-1}} \prod_{i \in I} \frac{x_{i}}{1-x_{i}},
$$

where $\left(\begin{array}{l}n \\ I\end{array}\right)_{X} \in \mathbb{Z}[X]$ denotes the Gaussian multinomial coefficient and

$$
x_{j}=q^{j(2 n-j)-s(n+j)} \text { for } j \in[n] .
$$

Remark 5.3. Let $k$ be a field. By a theorem of Schur, $\left(\begin{array}{cc}k \operatorname{Id}_{n} & \operatorname{Mat}_{n}(k) \\ 0 & k \operatorname{Id}_{n}\end{array}\right)$ is a maximal abelian subalgebra of $\operatorname{Mat}_{2 n}(k)$; cf. [28]. The right-hand side of (5.2) is a product of two Igusa functions in the terminology of [26, Definition 2.5].

Theorem 5.4. For all $g \in \mathbb{N}$ and $\mathbf{f}=\left(f_{1}, \ldots, f_{g}\right) \in \mathbb{N}^{g}$, the functional equation

$$
\left.\zeta_{\mathcal{M}_{\mathbf{f}}(\mathfrak{o}) \curvearrowright \mathfrak{o}^{2 n}}(s)\right|_{p \rightarrow p^{-1}}=q^{\left(\begin{array}{c}
2 n \\
2
\end{array}\right)-3 n s} \zeta_{\mathcal{M}_{\mathbf{f}}(\mathfrak{o}) \curvearrowright \mathfrak{o}^{2 n}}(s)
$$

holds.

Sketch of proof. Analogous to [26, Theorem 1.2] with $3 n$ and $5 n(=3 n+2 n)$ replaced by $2 n$ and $3 n(=2 n+n)$, respectively. 
5.2.2. Global zeta functions and Euler products. The local formulae in Section 5.2.1 may be put in a global context as follows. For any ring $A$, consider

$$
\mathcal{M}(A)=\left(\begin{array}{cc}
0 & A \\
0 & 0
\end{array}\right) \leq \operatorname{Mat}_{2}(A)
$$

Let $K$ be a number field with ring of integers $\mathcal{O}$ and of degree $n$, say. By restriction of scalars from $K$ to $\mathbb{Q}$, we may consider $\mathcal{M}(\mathcal{O})$ as a subalgebra of $\operatorname{Mat}_{2 n}(\mathbb{Z})$, turning $\mathbb{Z}^{2 n}$ into an $\mathcal{M}(\mathcal{O})$-module by right multiplication, with associated submodule zeta function

$$
\zeta_{\mathcal{M}(\mathcal{O}) \curvearrowright \mathbb{Z}^{2 n}}(s)=\prod_{p \text { prime }} \zeta_{\mathcal{M}\left(\mathcal{O} \otimes_{\mathbb{Z}} \mathbb{Z}_{p}\right) \curvearrowright \mathbb{Z}_{p}^{2 n}(s) .}
$$

This is a close analogue of the ideal zeta function $\zeta_{H(\mathcal{O})}^{\triangleleft}(s)$ of the Heisenberg Lie ring over $\mathcal{O}$ studied in [26]. If $p$ is unramified in $K$, i.e. $p \mathcal{O}=\prod_{i=1}^{g} \mathfrak{p}_{i}$ for pairwise distinct prime ideals $\mathfrak{p}_{i}$ of $\mathcal{O}$ with residue degrees $f_{i}$ for $i \in[g]$, then $\mathcal{O} \otimes_{\mathbb{Z}} \mathbb{Z}_{p} \cong \prod_{i=1}^{g} \mathbb{Z}_{p^{f_{i}}}$, where $\mathbb{Z}_{p^{f_{i}}}$ denotes the unramified extension of $\mathbb{Z}_{p}$ of degree $f_{i}$. Hence

$$
\mathcal{M}\left(\mathcal{O} \otimes_{\mathbb{Z}} \mathbb{Z}_{p}\right) \cong \mathcal{M}_{\mathbf{f}}\left(\mathbb{Z}_{p}\right) .
$$

Therefore, all but finitely many of the Euler factors in (5.3) are covered by the formulae sketched - and written out in Theorem 5.1 for the primes which split totally in $K$ and in Theorem 5.2 for the primes which stay inert in $K$ - in Section 5.2.1. Formulae for the Euler factors indexed by the rational primes which remain unsplit in $K$ (but may ramify) may be obtained by modifying those for $\zeta_{H(\mathcal{O})}^{\triangleleft}(s)$ described in [27]. Note that Theorem [1.2] is applicable for $\mathcal{E}=\mathcal{M}(\mathcal{O})$ and $\mathcal{L}=\mathbb{Z}^{2 n}$ as $c=2$; cf. Remark 1.8. The functional equations established in Theorem 5.4 strengthen the result in this special case by implying that the set of exceptional primes is contained in (and conjecturally equal to) the set of primes which ramify in $K$.

5.3. Strictly upper triangular matrices. For $m \in \mathbb{N}$, let $\mathfrak{u}_{m}(\mathfrak{o})$ be the associative algebra of all strictly upper triangular $m \times m$-matrices over $\mathfrak{o}$, acting on $\mathfrak{o}^{m}$ by rightmultiplication, say. Given a partition $\lambda=\left(\lambda_{1}, \ldots, \lambda_{r}\right) \in \mathbb{N}^{r}$ of $n=\sum_{i=1}^{r} \lambda_{i}$, consider

$$
\mathfrak{u}_{\lambda}(\mathfrak{o})=\bigoplus_{i=1}^{r} \mathfrak{u}_{\lambda_{i}}(\mathfrak{o}),
$$

diagonally embedded into $\mathfrak{u}_{n}(\mathfrak{o})$. Theorem 1.2 and Corollary 1.3 are clearly applicable and imply the following result.

Theorem 5.5. For almost all primes $p$ and all compact discrete valuation rings $\mathfrak{o}$ of residue characteristic $p$,

$$
\left.\zeta_{\mathfrak{u}_{\lambda}(\mathfrak{o}) \curvearrowright \mathfrak{o}^{n}}(s)\right|_{p \rightarrow p^{-1}}=(-1)^{n} q^{\left(\left(\begin{array}{c}
n \\
2
\end{array}\right)-s \sum_{i=1}^{r}\left(\begin{array}{c}
\lambda_{i}+1 \\
2
\end{array}\right)\right)} \zeta_{\mathfrak{u}_{\lambda}(\mathfrak{o}) \curvearrowright \mathfrak{o}^{n}}(s) .
$$

Explicit formulae for generic submodule zeta functions of the form $\zeta_{\mathfrak{u}_{\lambda}(\mathfrak{o}) \curvearrowright \mathfrak{o}^{n}}(s)$ have been computed for $\lambda=(m), m \leq 5$ as well as for numerous other partitions of natural numbers $n \leq 7$; see [24, Section 9.4] and the database in the computer algebra package 22. All these functions are given by rational functions in $q$ and $q^{-s}$. Theorem 5.5 is consistent with and explains the functional equations recorded in [24, Theorems 9.5, 9.7, $9.8]$. 


\section{ACKNOWLEDGMENTS}

I am grateful for helpful conversations I had with Luke Woodward, over and beyond the mathematical inspiration I gained from reading [13], a book whose influence on the paper I acknowledge in detail in Section 4.1. I thank Tobias Rossmann for numerous discussions which had a profound impact on this paper, rendering its results much more general and conceptual. I am grateful to two anonymous referees whose comments helped me to improve the paper's exposition significantly and prompted Corollary 1.3. To Raf Cluckers I am particularly endebted for elucidations about the transfer principle which greatly helped me in the formulation of this corollary. Work on this paper was supported by DFG Sonderforschungsbereich 701 at Bielefeld University.

\section{REFERENCES}

[1] N. Avni, B. Klopsch, U. Onn, and C. Voll, Representation zeta functions of compact p-adic analytic groups and arithmetic groups, Duke Math. J. 162 (2013), no. 1, 111-197.

[2] M. N. Berman, Uniformity and functional equations for local zeta functions of $\mathfrak{K}$-split algebraic groups, Amer. J. Math. 133 (2011), no. 1, 1-27.

[3] M. N. Berman and B. Klopsch, A nilpotent group without local functional equations for pro-isomorphic subgroups, J. Group Theory 18 (2015), no. 3, 489-510.

[4] M. N. Berman, B. Klopsch, and U. Onn, A family of class-2 nilpotent groups, their automorphisms and pro-isomorphic zeta functions, arXiv:1511.07418, 2015.

[5] _ On pro-isomorphic zeta functions of $D^{*}$-groups of even Hirsch length, arXiv:1511.06360, 2015.

[6] R. Cluckers and F. Loeser, Constructible exponential functions, motivic Fourier transform and transfer principle, Ann. of Math. (2) 171 (2010), no. 2, 1011-1065.

[7] J. Denef, On the degree of Igusa's local zeta function, Amer. J. Math. 109 (1987), no. 6, 991-1008.

[8] _ Report on Igusa's local zeta function, Séminaire Bourbaki 43 (1990-91), no. 201-203, 359-386.

[9] J. Denef and D. Meuser, A functional equation of Igusa's local zeta function, Amer. J. Math. 113 (1991), no. 6, 1135-1152.

[10] M. P. F. du Sautoy, The zeta function of $\mathfrak{s l}_{2}(\mathbf{Z})$, Forum Math. 12 (2000), no. 2, 197-221.

[11] M. P. F. du Sautoy and F. J. Grunewald, Analytic properties of zeta functions and subgroup growth, Ann. of Math. (2) 152 (2000), 793-833.

[12] M. P. F. du Sautoy and A. Lubotzky, Functional equations and uniformity for local zeta functions of nilpotent groups, Amer. J. Math. 118 (1996), no. 1, 39-90.

[13] M. P. F. du Sautoy and L. Woodward, Zeta functions of groups and rings, Lecture Notes in Mathematics, vol. 1925, Springer-Verlag, Berlin, 2008.

[14] A. Evseev, Reduced zeta functions of Lie algebras, J. Reine Angew. Math. 633 (2009), 197-211.

[15] P. Garrett, Buildings and classical groups, Chapman \& Hall, London, 1997.

[16] F. J. Grunewald, D. Segal, and G. C. Smith, Subgroups of finite index in nilpotent groups, Invent. Math. 93 (1988), 185-223.

[17] J.-I. Igusa, Complex powers and asymptotic expansions. I. Functions of certain types, J. Reine Angew. Math. 268/269 (1974), 110-130.

[18] N. Jacobson, Lie algebras, Dover Publications, Inc., New York, 1979.

[19] M. Hall Jr., A basis for free Lie rings and higher commutators in free groups, Proc. Amer. Math. Soc. 1 (1950), 575-581.

[20] A. Macintyre, Rationality of p-adic Poincaré series: uniformity in p, Ann. Pure Appl. Logic 49 (1990), no. $1,31-74$.

[21] T. Rossmann, Stability results for local zeta functions of groups, algebras, and modules, to appear in Math. Proc. Cambridge Philos. Soc.

[22] _ Zeta, http://www.math.uni-bielefeld.de/ rossmann/Zeta/, version 0.3.2.

[23] _ Computing topological zeta functions of groups, algebras, and modules, I, Proc. Lond. Math. Soc. (3) 110 (2015), no. 5, 1099-1134.

[24] _ Computing local zeta functions of groups, algebras, and modules, arxiv:1602.00919, 2016.

[25] — Enumerating submodules invariant under an endomorphism, Math. Ann. 368 (2017), 391-417.

[26] M. M. Schein and C. Voll, Normal zeta functions of the Heisenberg groups over number rings I - the unramified case, J. Lond. Math. Soc. (2) 91 (2015), no. 1, 19-46. 
[27] Normal zeta functions of the Heisenberg groups over number rings II - the non-split case, Israel J. Math. 211 (2016), 171-195.

[28] J. Schur, Zur Theorie der vertauschbaren Matrizen, J. Reine Angew. Math. 130 (1905), 66-76.

[29] L. Solomon, Zeta functions and integral representation theory, Adv. Math. 26 (1977), no. 3, 306-326.

[30] R. P. Stanley, Combinatorics and commutative algebra, Birkhäuser, 1996, second edition.

[31] _ Enumerative combinatorics, Cambridge Studies in Advanced Mathematics, 49, vol. 1, Cambridge University Press, 1997.

[32] A. Stasinski and C. Voll, Representation zeta functions of nilpotent groups and generating functions for Weyl groups of type B, Amer. J. Math. 136 (2014), no. 2, 501-550.

[33] C. Voll, Functional equations for local normal zeta functions of nilpotent groups, Geom. Funct. Anal. (GAFA) 15 (2005), 274-295, with an appendix by A. Beauville.

[34] _ Normal subgroup growth in free class-2-nilpotent groups, Math. Ann. 332 (2005), 67-79.

[35] Functional equations for zeta functions of groups and rings, Ann. of Math. (2) 172 (2010), no. $2,1181-1218$

[36] E. Witt, Treue Darstellung Liescher Ringe, J. Reine Angew. Math. 177 (1937), 152-160.

[37] L. Woodward, Zeta functions of Lie rings of upper-triangular matrices, J. Lond. Math. Soc. (2) 77 (2008), no. 1, 69-82.

Fakultät für Mathematik, Universität Bielefeld, Postfach 100131, D-33501 Bielefeld, GERMANY

E-mail address: C.Voll.98@cantab.net 